\title{
Electroacoustic Waves in a Ferroelectric Crystal with of a Moving System of Domain Walls
}

\author{
Vilkov E.A. and Maryshev S.N. \\ ${ }^{1}$ Kotel'nikov Institute of Radio Engineering and \\ Electronics of Russian Academy of Sciences, Ul'yanovsk Branch, \\ ${ }^{2}$ Moscow Institute of Physics and Technology \\ Russia
}

\section{Introduction}

In addition to conventional applications of ferroelectric crystals that exhibit piezoactivity, such crystals find wide application in the production of domain electronic devices based on the interaction between acoustic waves and ferroelectric domains (acousto-domain interaction) [Esayan et al, 1974]. In the course of the general development of technology of phonon crystals the ferroelectric with periodic domain structures (PDSs) have attracted a considerable interest recently [Golenishchev-Kutuzov et al, 2003]. The interest in such structures with periodically varying elastic, electric, and other physical parameters dates back to the 1960s since, by that time, the possibilities offered by single-domain crystals in the realization of the spectral characteristics of elastic and electromagnetic waves had been exhausted. First of all a ferroelectrics with PDSs differ from usual phonon crystals by ability of its reorganization under external action due to rather fast moving domain walls(DW) [Golenishchev-Kutuzov et al, 2003].

The spectra of elastic oscillations of a ferroelectric with a static PDS may significantly differ from the spectra of a conventional single-domain sample [Li, et al, 1991; Lyubimov \& Sannikov, 1979]. This difference is primarily manifested in the formation of forbidden and allowed bands, which are absent in the continuous spectrum of waves in a single-domain crystal. To the full this conclusion extends to oscillation modes of localized at DWs, in particular - to electroacoustic interfacial waves (EIWs) [Maerfield \& Tournois, 1971], which were widely studied in ferroelectrics with static PDSs, representing a superlattice [Li, et al, 1991; Nougaoui, 1987]. Considerable modification of spectral parameters of EIWs in superlattices such as boundary localization of oscillation and phase speed was explained in [Lyubimov \& Sannikov, 1979; Li, 1987] as result of interaction between electrosound oscillations of neighbo(u)ring DW.

At an early stage of research the possibility of the realization of PDSs with various periods of the lattices of domain walls and the possibility of vary lattice period [GolenishchevKutuzov et al, 2003] have shown the prospects of application of the domain superlattices. It was found out, in particular, that crystals with PDSs are effective as broadband piezoconverters of acoustic waves, and with the greater efficiency of transformation, than have monodomain crystals [Batanova \& Golenishchev-Kutuzov, 1997]. Other fields of application (the acoustic spectroscopy of polydomain ferroelectrics and processing of the 
signal information) have demanded of studying of effects of a refraction of acoustic waves from packages of DWs of ferroelectrics. So, in development of results of work [Esayan et al, 1974] it has been shown, that appropriate variations in the period of the domain lattice, crystal parameters, and conditions of wave propagation make it possible to control the height, number, and location of Bragg reflections peaks [Shuvalov \& Gorkunova, 1999; Shenderov,1997], thus providing prospects for various applications.

All the results listed above are related to the static case, i.e. they are obtained for domain lattices with motionless DW. It means that the manipulation of PDSs expressed by moving DWs occurs not during the process of acousto-domain interaction. The overlap of the procedure of adjustment PDSs and the process of acousto-domain interaction is interesting due to a number of circumstances.

First, this overlap demands modeling of a supernumerary situation of work of the acoustodomain device at "failure" of device domain structure. Second, it is necessary for research of additional opportunities, for example, scanning of a crystal by means of EIWs on moving DW [Gulyaev et al, 2000] or realization of ideas put forward by Auld [Auld,1973] of the Doppler transformation of a spectrum of frequencies of bulk acoustic waves refracted by moving DW [Shevyakhov, 1990]. Thus, it is expedient to generalize the results of the research of acoustic properties of static superlattices of ferroelectric to the case of dynamic superlattices whose distinctive feature is adjustable moving domain boundaries. Below, on the basis of our works [Vilkov, 2008; Vilkov, 2009; Vilkov et al, 2009] the propagation of guided EIWs and bulk electrosound waves in a dynamic superlattice of equidistant uniformly moving 180-degree DW is considered. Let's notice, that until now the research of acoustic effects in crystals of ferroelectrics with moving DW was limited to cases of noncollective acousto-domain interactions:a single DW [Shevyakhov, 1990; Gulyaev et al, 2000], a strip domain (i.e., a pair of DWs) [El'meshkin \& Shevyakhov, 2006] and a structure of a two adjacent strip domains [Bugaev et al, 2005].

\section{Basic equations for a shear wave in ferroelectric in the frame of the DW at rest}

In acoustoelectronics, the elastic deformation and electric-field intensity $E_{j}$ normally serve as independent quantities that determine the state of a piezoactive ferroelectric medium. The elastic deformation is determined by the deformation tensor

$$
u_{i k}=\frac{1}{2}\left(\frac{\partial u_{i}}{\partial x_{k}}+\frac{\partial u_{k}}{\partial x_{i}}\right),
$$

For the adiabatic deformation, the equations of state are written as follows [Sosnin \& Strukov, 1970]

$$
\begin{gathered}
T_{i k}=c_{i k l m} u_{l m}-e_{j, i k} E_{j} \\
D_{p}=\varepsilon_{p q} E_{q}+4 \pi e_{p, r m} u_{r m}
\end{gathered} .
$$

where $T_{i k}$ is the mechanical stress tensor, which is a second- rank tensor (as well as deformation tensor $u_{i k}$ given by expression (1)), and $c_{i k l m}, e_{j, i k}$ and $\varepsilon_{p q}$ are the tensors of elastic, piezoelectric, and dielectric moduli of a crystal. Note that summation over the 
repeated subscripts is involved in Eq. (2), where $D_{p}$ is the electric induction. The initial equations are the equation of motion for a crystal

$$
\rho \frac{\partial^{2} u_{i}}{\partial t^{2}}=\frac{\partial T_{i k}}{\partial x_{k}}
$$

and the Maxwell equations in the quasi-static representation,

$$
E_{i}=-\frac{\partial \varphi}{\partial x_{i}}, \quad \frac{\partial D_{k}}{\partial x_{k}}=0 .
$$

Here, differential operator $\partial / \partial x_{i}$ is considered a vector, so that tensor summation over repeated subscript $k$ is involved in the second line of expression (4); $\varphi$ is the electric potential; and $\rho$ is the crystal density.

For the consistent analysis of Eqs. (1)-(4), we specify the symmetry of the crystal and the propagation configuration of acoustic waves. We assume that, $4 \mathrm{~mm}$ ferroelectric has a crystallographic putting such that, in a crystallographic system the fourth-order symmetry axis that is parallel to the $z$ axis of laboratory frame $x O y z$. For the given crystal symmetry initial Eqs. (1)-(4) can be presented after some transformations in the form of

$$
\begin{aligned}
& 4 \pi e_{1,5}\left(\frac{\partial^{2} u_{z}}{\partial x^{2}}+\frac{\partial^{2} u_{z}}{\partial y^{2}}\right)+4 \pi e_{3,3} \frac{\partial^{2} u_{z}}{\partial z^{2}}+4 \pi\left(e_{1,5}+e_{3,1}\right) \frac{\partial}{\partial z}\left(\frac{\partial u_{x}}{\partial x}+\frac{\partial u_{y}}{\partial y}\right)=\varepsilon_{1}\left(\frac{\partial^{2} \varphi}{\partial x^{2}}+\frac{\partial^{2} \varphi}{\partial y^{2}}\right)+\varepsilon_{3} \frac{\partial^{2} \varphi}{\partial z^{2}} \\
& \rho \frac{\partial^{2} u_{x}}{\partial t^{2}}=c_{11} \frac{\partial^{2} u_{x}}{\partial x^{2}}+c_{66} \frac{\partial^{2} u_{x}}{\partial y^{2}}+c_{44} \frac{\partial^{2} u_{x}}{\partial z^{2}}+\left(c_{12}+c_{66}\right) \frac{\partial^{2} u_{y}}{\partial x \partial y}+\left(c_{13}+c_{44}\right) \frac{\partial^{2} u_{z}}{\partial x \partial z}+\left(e_{1,5}+e_{3,1}\right) \frac{\partial^{2} \varphi}{\partial x \partial z} \\
& \rho \frac{\partial^{2} u_{y}}{\partial t^{2}}=c_{66} \frac{\partial^{2} u_{y}}{\partial x^{2}}+c_{11} \frac{\partial^{2} u_{y}}{\partial y^{2}}+c_{44} \frac{\partial^{2} u_{y}}{\partial z^{2}}+\left(c_{12}+c_{66}\right) \frac{\partial^{2} u_{x}}{\partial x \partial y}+\left(c_{13}+c_{44}\right) \frac{\partial^{2} u_{z}}{\partial y \partial z}+\left(e_{1,5}+e_{3,1}\right) \frac{\partial^{2} \varphi}{\partial y \partial z} \\
& \rho \frac{\partial^{2} u_{z}}{\partial t^{2}}=c_{44}\left(\frac{\partial^{2} u_{z}}{\partial x^{2}}+\frac{\partial^{2} u_{z}}{\partial y^{2}}\right)+c_{33} \frac{\partial^{2} u_{z}}{\partial z^{2}}+\left(c_{13}+c_{44}\right)\left(\frac{\partial^{2} u_{x}}{\partial x \partial z}+\frac{\partial^{2} u_{y}}{\partial y \partial z}\right)+e_{1,5}\left(\frac{\partial^{2} \varphi}{\partial x^{2}}+\frac{\partial^{2} \varphi}{\partial y^{2}}\right)+e_{3,3} \frac{\partial^{2} \varphi}{\partial z^{2}} .
\end{aligned}
$$

The received system of the equations (6) together with the equation (5) describes dynamic behaviour of a piezoelectric material of a class $4 \mathrm{~mm}(6 \mathrm{~mm}, \infty \mathrm{m})$ and often is called as system of the equations of piezoacoustics. We assume also that, the propagation of acoustic waves is in the direction perpendicular to the high-order symmetry axis. In these conditions the solution is not depend on coordinate $\mathrm{z}$ and the system of the equations (6) are split into two independent systems of equations. First of them

$$
\begin{aligned}
& \rho \frac{\partial^{2} u_{x}}{\partial t^{2}}=c_{11} \frac{\partial^{2} u_{x}}{\partial x^{2}}+c_{66} \frac{\partial^{2} u_{x}}{\partial y^{2}}+\left(c_{12}+c_{66}\right) \frac{\partial^{2} u_{y}}{\partial x \partial y}, \\
& \rho \frac{\partial^{2} u_{y}}{\partial t^{2}}=c_{66} \frac{\partial^{2} u_{y}}{\partial x^{2}}+c_{11} \frac{\partial^{2} u_{y}}{\partial y^{2}}+\left(c_{12}+c_{66}\right) \frac{\partial^{2} u_{x}}{\partial x \partial y},
\end{aligned}
$$

and describe horizontally polarized waves: $\mathbf{u} \perp z$. The second system added by the equation (5) 


$$
\begin{gathered}
\rho \frac{\partial^{2} u_{z}}{\partial t^{2}}=c_{44}\left(\frac{\partial^{2} u_{z}}{\partial x^{2}}+\frac{\partial^{2} u_{z}}{\partial y^{2}}\right)+e_{1,5}\left(\frac{\partial^{2} \varphi}{\partial x^{2}}+\frac{\partial^{2} \varphi}{\partial y^{2}}\right), \\
4 \pi e_{1,5}\left(\frac{\partial^{2} u_{z}}{\partial x^{2}}+\frac{\partial^{2} u_{z}}{\partial y^{2}}\right)=\varepsilon_{1}\left(\frac{\partial^{2} \varphi}{\partial x^{2}}+\frac{\partial^{2} \varphi}{\partial y^{2}}\right) .
\end{gathered}
$$

describes horizontally polarized waves: $\mathbf{u}|| z$.

Waves of horizontal and vertical polarization do not interact among themselves. At the same time, elastic displacements in vertically polarized waves are not accompanied by the generation of the electric field, so that the crystal behaves toward these waves as a conventional elastic medium in the absence of the piezoelectric effect. In the further analysis, we disregard vertically polarized waves owing to the absence of the acousto-domain interaction. Horizontally polarized waves with the particle displacements along the $z$ axis are piezoelectrically active and accompanied by the electric field in the propagation plane. (see Fig. 1).

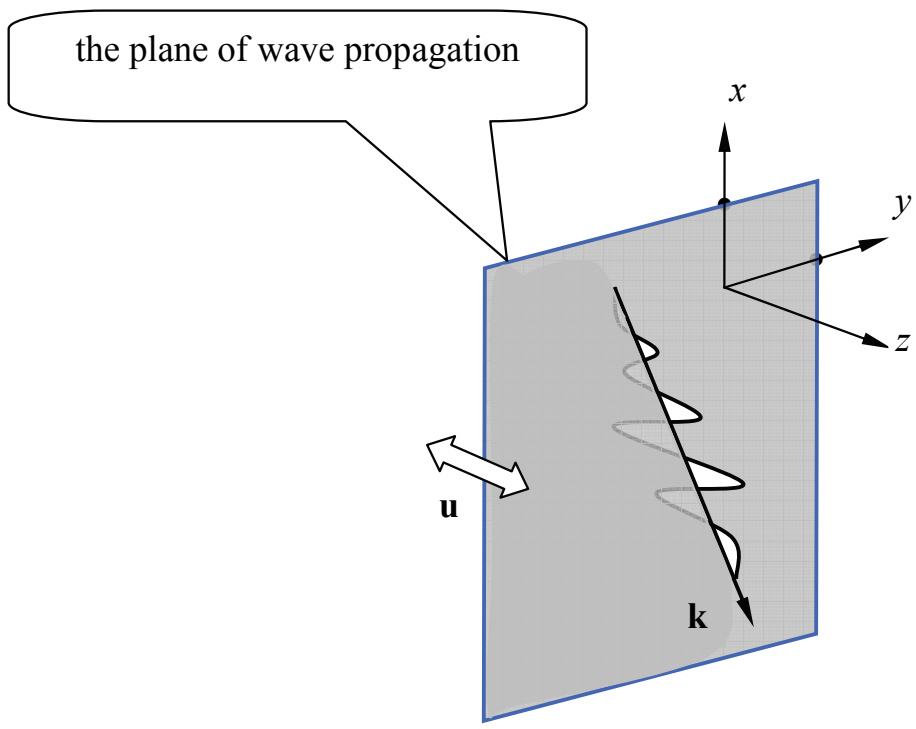

Fig. 1. The geometry of the propagation of a shift wave of horizontal polarization

For final writing of the equations (8) we consider, that the piezoelectric modulus changes the sign in the neighboring domains divided by 180-degree DWs because of the antiparallel orientation of the polar directions, i.e. the plane of wave propagation

$$
e^{(j)}=(-1)^{j+1} e_{15}, \quad e_{15}>0 .
$$

where $j=1$ and 2 stand for domains of the " + " and "-" types with positive and negative piezoelectric moduli, respectively. As a result we write down of the equation (8) in the form of

$$
\rho \frac{\partial^{2} u_{j}}{\partial t^{2}}=c_{44} \nabla^{2} u_{j}+e^{(j)} \nabla^{2} \varphi_{j}, \quad 4 \pi e^{(j)} \nabla^{2} u_{j}=\varepsilon_{1} \nabla^{2} \varphi_{j}
$$


where $\nabla^{2}=\left(\partial^{2} / \partial x^{2}+\partial^{2} / \partial y^{2}\right)$ is the Laplace operator, and shift displacement $u_{z}$ have designated as $u_{j}$ according number of domain $\mathrm{j}$.

Domain walls can move in ferroelectrics under specific external actions. In this case, to construct a solution in domains, it is necessary to consider the equation (10) in the frame of the DW at rest $\tilde{x} 0 \tilde{y} \tilde{z}$. Because $V_{D} \ll c\left(c\right.$ is the velocity of light, $V_{D}$ is the velocity of DW), we employ the Galilean transformation for the connection of coordinates:

$$
\tilde{x}=x, \quad \tilde{y}=y-V_{D} t, \quad \tilde{t}=t .
$$

Using the relationships between the differential operators according to (11):

$$
\frac{\partial}{\partial y} \leftrightarrow \frac{\partial}{\partial \tilde{y}}, \quad \frac{\partial}{\partial x} \leftrightarrow \frac{\partial}{\partial \tilde{x}}, \quad \frac{\partial}{\partial t} \leftrightarrow \frac{\partial}{\partial \tilde{t}}-V_{D} \frac{\partial}{\partial \tilde{y}}
$$

and expressions (11) and (12), we can easily represent Eqs. (10) as

$$
\left[\frac{1}{c_{44}^{* 2}}\left(\frac{\partial}{\partial \tilde{t}}-V_{D} \frac{\partial}{\partial \tilde{y}}\right)^{2}-\tilde{\nabla}^{2}\right] u_{j}=0, \quad \tilde{\nabla}^{2} \Phi_{j}=0
$$

In these equations, $c_{44}^{*}=c_{44}+4 \pi e_{15}^{2} / \varepsilon_{1}$, and $\Phi j$ is the component of total potential $\varphi_{j}$ in the jth domain,

$$
\varphi_{j}=-\frac{4 \pi e^{(j)}}{\varepsilon_{1}} u_{j}+\Phi_{j}
$$

which corresponds to the electric field induced from the boundaries $\tilde{y}_{n}=y_{n}-V_{D} t$ by piezopolarization charges [Lyubimov \& Sannikov, 1979].

\section{The electroacoustic bulk propagating waves in a ferroelectric with a system of moving periodic domain structure}

\subsection{The statement of the problem}

In this section we consider the influence of a uniform motion of domain boundaries forming a dynamic superlattice of the tetragonal ferroelectric, on spectral properties of bulk electrosound waves (EW). The comparison of the phonon spectra of static and dynamic superlattices allows a more detailed description of specific wave processes realized in periodic structures. The results obtained indicate that even slow DW motions cause significant (detectable) variations in the wave spectra.

The schematic of the problem is presented in Fig. 2. We assume that, in a crystallographic system with the fourth-order symmetry axis that is parallel to the $z$ axis of laboratory frame $x O y z$, a $4 m m$ ferroelectric represents a superlattice of 180 -degree DWs with period $2 d(d>>\Delta$ is the distance between neighboring DWs and $\Delta$ is the DW thickness). The superlattice uniformly moves at the velocity $V_{D}|| y[010] \|$. Positive velocity $V_{D}$ corresponds to the superlattice motion codirectional with the $y$ axis. To eliminate the significant structural sensitivity of DWs to the regime of motion related to the generation of both spontaneous polarization and spontaneous deformation in domains [Sosnin. \& Strukov, 1970; Vainshtein, 
1988], we restrict the consideration to subsonic DW velocities. We also assume that the ferroelectric is far from the phase transition. Then, the DW motion is realized at $V_{D}=$ const and the current coordinates are given by $y_{n}=V_{D} t+n d$, where $t$ is time; $\mathrm{n}=0, \pm 1, \pm 2, \ldots$ and DWs are geometrically thin and structureless objects $(\mathrm{k} \Delta<<1$, where $k$ is the EW wave number).

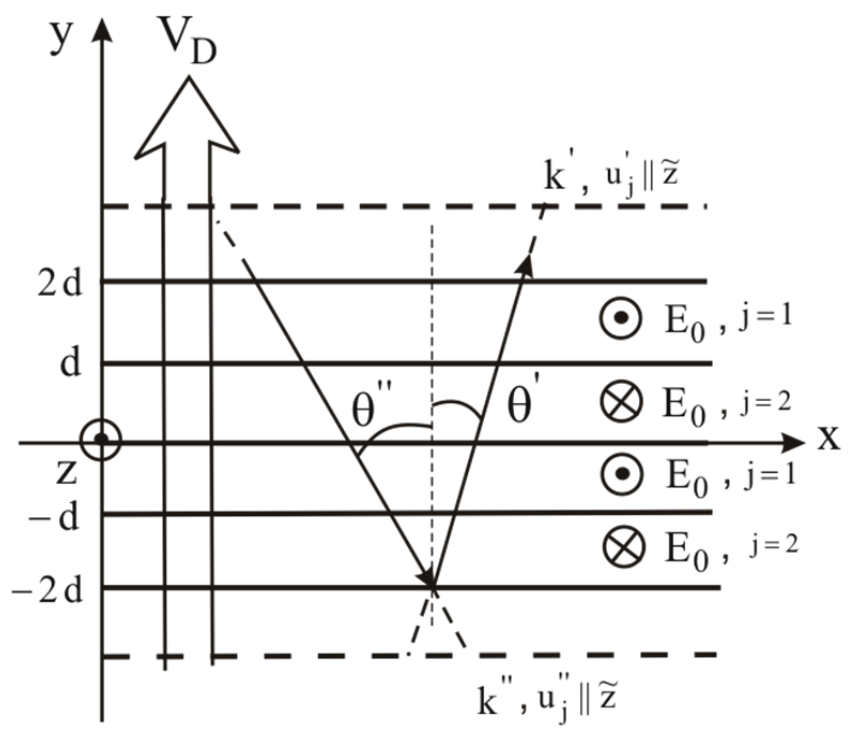

Fig. 2. Schematic representation of a domain superlattice with an electroacoustic eigenwave. The dashed lines indicate that two elementary cells are reproduced in the positive and negative directions of the $Y$ axis and form an infinite superlattice. Vectors k' and 'k"show the direction of the EW propagation. The arrow shows the direction of the lattice motion. Here, $\mathrm{E}_{0}$ is the electric-field intensity in domains

It is known [Vainshtein, 1988] that the above conditions are valid for DWs in $\mathrm{BaTiO}_{3}$-type ferroelectrics at ultrasonic frequencies. It is commonly accepted [Maerfield \& Tournois, 1971; Auld,1973] that, in this case, the ferroelectric can be considered as a piezoelectric crystal that is periodically twinned with respect to the planes $y=y_{n}$. With regard to the analysis of propagation of only horizontally polarized shear waves in the (001) crystal plane, we assume that antiparallel polarizations in the neighboring domains are related to the sign alternation of the piezoelectric modulus according equation (9). At that, there are in eq.(9) $j=1$ for $(n-1) d+V_{D} t<y<n d+V_{D} \mathrm{t}$ and $\mathrm{j}=2$ for $n d+V_{D} t<y<(n+1) d+V_{D} t(n=0, \pm 2, \pm 4, \ldots)$. The remaining characteristics of domains are identical.

Then, we assume that the electroacoustic eigenwaves with the wave vector $=k^{\prime}=\left(k^{\prime} \sin \theta^{\prime}\right.$, $\left.\mathrm{k}^{\prime} \cos \theta^{\prime}, 0\right)$ propagate in the XOY plane at angle $\theta^{\prime}$ relative to the normal to the plane of 180degree DWs in the positive direction of the $y$ axis (Fig. 2). For the electroacoustic eigenwaves propagating in the XOY plane at angle $\theta^{\prime \prime}$ to the normal to the DW plane in the negative direction of the $y$ axis, we employ another wave vector, $=\mathrm{k}^{\prime \prime}=\left(\mathrm{k}^{\prime \prime} \sin \theta^{\prime \prime}, \mathrm{k}^{\prime \prime} \cos \theta^{\prime \prime}, 0\right)$ in accordance with another expected Doppler shift [Shevyakhov, 1990]. 
The system of equations for these waves in the neighboring domains with numbers $j=1$ and 2, which results from the reduction of Eqs. (3) and (4) in accordance with the cyclic Bloch conditions in the case of 180-degree DWs, is represented by equations (9),(13), (14). The fields on the moving DWs of the lattice exhibit the phase conjugation for the wave characteristics of EWs that travel in the direction opposite to that of the DW propagation. Hence, the following relationships are valid [Shevyakhov, 1990]:

$$
\cos \theta^{\prime}=\frac{\cos \theta^{\prime \prime}\left(1+V_{D}^{2} / v^{2}\right)+2 V_{D} / v}{1+2\left(V_{D} / v\right) \cos \theta^{\prime \prime}+V_{D}^{2} / v^{2}}, \quad k_{y}^{\prime}=-k_{y}^{\prime \prime}+\frac{2 k_{y}^{\prime \prime}+2\left(V_{D} / v\right) k^{\prime \prime}}{1-V_{D}^{2} / v^{2}} .
$$

Here, $v$ is the EW phase velocity in a single-domain crystal. In the case of a static lattice $\left(V_{D}=0\right)$, the wave characteristics of the oppositely directed eigenwaves are identical: $\theta=\theta^{\prime \prime}=\theta^{\prime}, k_{y}=k_{y}^{\prime \prime}=k_{y}^{\prime}$.

To ensure the interaction between an EW with wave vector $\mathrm{k}^{\prime}$ and a DW moving away, we must provide for faster (in the signal meaning) wave propagation relative to the DW. In accordance with [Shevyakhov, 1990], this can be realized through imposition of a limitation on propagation angle $\theta^{\prime}, \theta^{\prime}<\theta^{* *}$. Owing to the coincidence of the phase and group velocities of an EW in a singledomain crystal, the critical angle $\theta^{* *}=\operatorname{arcos}\left(-V_{D} / v\right)$ determines the equality of the projection of the EW group velocity on the direction of the DW motion and the DW velocity.

When $\theta^{\prime}>\theta^{* *}$, the DW motion along the direction of the DW displacement is faster than the motion of any plane train of EWs with the same angle of incidence $\theta^{\prime}$. From the physical point of view, this situation corresponds to the vanishing acousto-domain interaction. In addition to the condition $\theta^{\prime}<\theta^{* *}$, we assume that the EW wavelength is significantly smaller than the crystal dimension. Under such conditions, the boundary effects at the outer interfaces of the ferroelectric and its shape weakly affect the behavior of waves and, hence, can be disregarded.

\subsection{Dispersion equation}

We assume that the domains with numbers 1 and 2 are located between the DWs with the coordinates $\tilde{y}=0, d, 2 d$ (Fig. 2). We search for the solution to Eqs. (13) in the region of the first domain in the following representation:

$$
\begin{aligned}
& u_{1}(\tilde{y})=\left(A_{1} \exp \left(i k_{y}^{\prime \prime} \tilde{y}\right)+A_{2} \exp \left(-i k_{y}^{\prime} \tilde{y}\right)\right) \exp \left(i\left(k_{x} \tilde{x}-\Omega \tilde{t}\right)\right), \\
& \Phi_{1}(\tilde{y})=\left(C_{1} \exp \left(k_{x} \tilde{y}\right)+C_{2} \exp \left(-k_{x} \tilde{y}\right)\right) \exp \left(i\left(k_{x} \tilde{x}-\Omega \tilde{t}\right)\right)
\end{aligned}
$$

Similar expressions can be written in the region of the second domain:

$$
\begin{aligned}
& u_{2}(\tilde{y})=\left(B_{1} \exp \left(i k_{y}^{\prime \prime} \tilde{y}\right)+B_{2} \exp \left(-i k_{y}^{\prime} \tilde{y}\right)\right) \exp \left(i\left(k_{x} \tilde{x}-\Omega \tilde{t}\right)\right) \\
& \Phi_{2}(\tilde{y})=\left(D_{1} \exp \left(k_{x} \tilde{y}\right)+D_{2} \exp \left(-k_{x} \tilde{y}\right)\right) \exp \left(i\left(k_{x} \tilde{x}-\Omega \tilde{t}\right)\right) .
\end{aligned}
$$

To join the domain fields in the frame of the DW at rest on the interface $\tilde{y}=d$ in the nonrelativistic quasistatic approximation, we employ the conventional continuity conditions [Auld, 1973] for shear displacements; potentials; the shear components of the stress tensor, 


$$
T_{y z}^{(j)}=c_{44} \frac{\partial u_{j}}{\partial \tilde{y}}-e^{(j)} \frac{\partial \varphi_{j}}{\partial \tilde{y}}
$$

and the $y$ components of the electric induction,

$$
D_{y}^{(j)}=\varepsilon_{1} \frac{\partial \varphi_{j}}{\partial \tilde{y}}-4 \pi e^{(j)} \frac{\partial u_{j}}{\partial \tilde{y}} .
$$

Thus, with allowance for expressions (14), (18), and (19), we obtain the following boundary conditions:

$$
\begin{aligned}
& \varphi_{1}(\tilde{y})=\varphi_{2}(\tilde{y}), \quad \frac{\partial \Phi_{1}}{\partial \tilde{y}}=\frac{\partial \Phi_{2}}{\partial \tilde{y}}, \quad u_{1}=u_{2} \\
& c_{44}^{*} \frac{\partial u_{1}}{\partial \tilde{y}}+e_{15} \frac{\partial \Phi_{1}}{\partial \tilde{y}}=c_{44}^{*} \frac{\partial u_{2}}{\partial \tilde{y}}-e_{15} \frac{\partial \Phi_{2}}{\partial \tilde{y}},
\end{aligned}
$$

To simplify the further analysis, we introduce the following quantities:

$$
\sigma_{1,2}(\tilde{y})=\frac{\partial \Phi_{1,2}}{\partial \tilde{y}}, \quad \alpha_{1,2}(\tilde{y})=c_{44}^{*} \frac{\partial u_{1,2}}{\partial \tilde{y}} \pm e_{15} \frac{\partial \Phi_{1,2}}{\partial \tilde{y}} .
$$

In the expressions for $\mathbf{u}_{1,2}, \alpha_{1,2}, \varphi_{1,2}$, and $\sigma_{1,2}$ we represent arbitrary constants $A_{1,2}, B_{1,2}, C_{1,2}$, $\mathrm{D}_{1,2}$ in terms of their values on the boundary $\tilde{y}=0$ (for the domain layer with the subscript $j=1$ ) and on the boundary $\tilde{y}=d$ (for the domain layer with the subscript $j=2$ ) and substitute these representations into expressions (14),(16), (17) and (21). The resulting formulas are as follows:

$$
\begin{gathered}
u_{1,2}(\tilde{y})=m u_{1,2}+\frac{m_{1}}{c_{44}^{*}\left(k_{y}^{\prime \prime}+k_{y}^{\prime}\right)} \alpha_{1,2}+0 \varphi_{1,2} \pm \frac{e_{15} m_{1}}{c_{44}^{*}\left(k_{y}^{\prime \prime}+k_{y}^{\prime}\right)} \sigma_{1,2} \\
\alpha_{1,2}(\tilde{y})=-\left(\frac{c_{44}^{*} k_{y}^{\prime \prime} k_{y}^{\prime} m_{1}}{\left(k_{y}^{\prime \prime}+k_{y}^{\prime}\right)}+c_{44}^{*} K^{2} k_{x} \operatorname{sh}\left(k_{x} \tilde{y}\right)\right) u_{1,2}+m^{\prime} \alpha_{1,2} \pm e_{15} k_{x} \operatorname{sh}\left(k_{x} \tilde{y}\right) \varphi_{1,2} \pm e_{15}\left(\operatorname{ch}\left(k_{x} \tilde{y}\right)-m\right) \sigma_{1,2} \\
\varphi_{1,2}(\tilde{y})= \pm \frac{c_{44}^{*} K^{2}\left(m-\operatorname{ch}\left(k_{x} \tilde{y}\right)\right)}{e_{15}} u_{1,2} \pm \frac{K^{2} m_{1}}{e_{15}\left(k_{y}^{\prime \prime}+k_{y}^{\prime}\right)} \alpha_{1,2}+\operatorname{ch}\left(k_{x} \tilde{y}\right) \varphi_{1,2}+\left(-\frac{K^{2} m_{1}}{\left(k_{y}^{\prime \prime}+k_{y}^{\prime}\right)}+\frac{\operatorname{sh}\left(k_{x} \tilde{y}\right)}{k_{x}}\right) \sigma_{1,2} \\
\sigma_{1,2}(\tilde{y})= \pm\left(-\frac{c_{44}^{*} K^{2} k_{x}}{e_{15}}\right) \operatorname{sh}\left(k_{x} \tilde{y}\right) u_{1,2}+0 \alpha_{1,2}+k_{x} \operatorname{sh}\left(k_{x} \tilde{y}\right) \varphi_{1,2}+\operatorname{ch}\left(k_{x} \tilde{y}\right) \sigma_{1,2}
\end{gathered}
$$

where the values of the fields $u_{1,2}, \alpha_{1,2}, \varphi_{1,2}$, and $\sigma_{1,2}$ on the right-hand side of the identities for the subscript $j=1$ correspond to $\tilde{y}=0$ and the plus sign is selected. For the subscript $j=$ 2 , we use the field values at $\tilde{y}=d$ and choose the minus sign. In expressions (22), we introduce quantity $K^{2}=4 \pi e_{15}^{2} / \varepsilon_{1} c_{44}^{*}$, which is the squared coefficient of the electromechanical coupling of the crystal for the shear waves that propagate in the (001) basis plane. For brevity, we use the following notation in expressions (22): 


$$
\begin{gathered}
m^{\prime}=2 \frac{\left(k_{y}^{\prime \prime} k_{y}^{\prime}\right)^{1 / 2}}{\left(k_{y}^{\prime \prime}+k_{y}^{\prime}\right)} \cos \left[\frac{\left(k_{y}^{\prime \prime}+k_{y}^{\prime}\right) \tilde{y}}{2}+i \ln \left(\frac{k_{y}^{\prime \prime}}{k_{y}^{\prime}}\right)^{\frac{1}{2}}\right] \exp \left[i \frac{\left(k_{y}^{\prime}-k_{y}^{\prime \prime}\right) \tilde{y}}{2}\right] \\
m=2 \frac{\left(k_{y}^{\prime \prime} k_{y}^{\prime}\right)^{1 / 2}}{\left(k_{y}^{\prime \prime}+k_{y}^{\prime}\right)} \cos \left[\frac{\left(k_{y}^{\prime \prime}+k_{y}^{\prime}\right) \tilde{y}}{2}+i \ln \left(\frac{k_{y}^{\prime}}{k_{y}^{\prime \prime}}\right)^{\frac{1}{2}}\right] \exp \left[i \frac{\left(k_{y}^{\prime}-k_{y}^{\prime \prime}\right) \tilde{y}}{2}\right], \\
m_{1}=2 \sin \left[\frac{\left(k_{y}^{\prime \prime}+k_{y}^{\prime}\right) \tilde{y}}{2}\right] \exp \left[i \frac{\left(k_{y}^{\prime}-k_{y}^{\prime \prime}\right) \tilde{y}}{2}\right] .
\end{gathered}
$$

Thus, for $V_{D}=0$, , we have $m=\cos \left(k_{y} \tilde{y}\right)$ and $m_{1}=2 \sin \left(k_{y} \tilde{y}\right)$ with allowance for expression (15).

We represent the fields in the domain with the subscript $j=1$ on the DW $\tilde{y}=0$ in terms of their values at arbitrary point $\tilde{y}$ of the given layer. For the domain with the subscript $j=2$, we represent the fields on the DW $\tilde{y}=d$ in terms of their values at an arbitrary point of this layer. For this purpose, we find matrices that are inverses of the $4 \times 4$ square matrices consisting of the coefficients of $\mathbf{u}_{1,2}, \alpha_{1,2}, \varphi_{1,2}$ and $\sigma_{1,2}$ in expression (18). At $\tilde{y}=d$, these inverse matrices are represented as

$$
\mathbf{M}_{j}(d)=\left(\begin{array}{cccc}
\frac{m}{\delta} & -\frac{m_{1}}{c_{44}^{*}\left(k_{y}^{\prime \prime}+k_{y}^{\prime}\right) \delta} & 0 & \frac{e_{j} m_{1}}{c_{44}^{*}\left(k_{y}^{\prime \prime}+k_{y}^{\prime}\right) \delta} \\
\frac{c_{44}^{*} k_{y}^{\prime \prime} k_{y}^{\prime}}{\left(k_{y}^{\prime \prime}+k_{y}^{\prime}\right) \delta} m_{1}+K^{2} \mu^{*} k_{x} \operatorname{sh}\left(k_{x} d\right) & \frac{m^{\prime}}{\delta} & -e_{j} \operatorname{sh}\left(k_{x} d\right) & e_{j}\left(-m / \delta+\operatorname{ch}\left(k_{x} d\right)\right) \\
\frac{c_{44}^{*} K^{2}}{e_{j}}\left(m / \operatorname{det}-\operatorname{ch}\left(k_{x} d\right)\right) & \frac{-K^{2} m_{1}}{e_{j}\left(k_{y}^{\prime \prime}+k_{y}^{\prime}\right) \delta} & \operatorname{ch}\left(k_{x} d\right) & \frac{-K^{2} m_{1}}{e_{j}\left(k_{y}^{\prime \prime}+k_{y}^{\prime}\right) \delta}-\frac{\operatorname{sh}\left(k_{x} d\right)}{k_{x}} \\
\frac{c_{44}^{*} K^{2} k_{x} \operatorname{sh}\left(k_{x} d\right)}{e_{j}} & 0 & -k_{x} \operatorname{sh}\left(k_{x} d\right) & \operatorname{ch}\left(k_{x} d\right)
\end{array}\right)
$$

where $\delta=m m^{\prime}+\left(k_{y}^{\prime} k_{y}^{\prime \prime} m_{1}^{2}\right) /\left(k_{y}^{\prime}+k_{y}^{\prime \prime}\right)^{2}$. The matrices $\mathbf{M}_{1}=\mathbf{M}_{1}(d)$ and $\mathbf{M}_{2}=\mathbf{M}_{2}(d)$, which establish the relationships between the fields at the beginning and end of a domain layer, are called transition matrices [Bass, 1989]. It is known [Bass, 1989] that the product $\mathbf{M}=\mathbf{M}_{1} \cdot \mathbf{M}_{2}$ determines the transition matrix on the period of the structure. We do not present the elements of this matrix, since the corresponding expressions are cumbersome even for a static superlattice.

Using the transition matrix, we derive a system of algebraic equations to find the dispersion equation. This system is two times smaller than that obtained according to the conventional approach from [Bass, 1989]. To find the dispersion equation for an infinite structure, we need to determine the eigenvalues $\lambda=\exp (2 i \kappa d)$ of matrix $\mathbf{M}$. For this purpose, we must find and set to zero the determinant $\operatorname{Det}[\mathbf{M}-\lambda \mathbf{E}]=0$, where $\mathbf{E}$ is the $4 \times 4$ unit matrix and $\kappa$ is the Bloch vector, which represents the transverse wave number averaged over the period of the 
structure. The corresponding transformations yield the EW dispersion equation in the case of a moving superlattice:

$$
\begin{aligned}
& \lambda^{4}+\lambda^{3}\left[\frac{-\left(m^{2}+m^{\prime 2}\right)+}{\delta^{2}}+\frac{2}{\delta^{2}} \frac{k_{y}^{\prime} k_{y}^{\prime \prime} m_{1}^{2}}{\left(k_{y}^{\prime \prime}+k_{y}^{\prime}\right)^{2}}-2 \operatorname{ch}\left(2 k_{x} d\right)+\frac{8 K^{2} k_{x} m_{1} \operatorname{sh}\left(k_{x} d\right)}{\delta\left(k_{y}^{\prime \prime}+k_{y}^{\prime}\right)}\right]+ \\
& +\lambda^{2}\left[1+\frac{1}{\delta^{2}}+\frac{16 K^{4} k_{x}^{2} m_{1}^{2}}{\delta^{2}\left(k_{y}^{\prime \prime}+k_{y}^{\prime}\right)^{2}} \operatorname{sh}^{2}\left(k_{x} d\right)+\frac{2 \operatorname{ch}\left(2 k_{x} d\right)}{\delta^{2}}\left(\left(m^{2}+m^{\prime 2}\right)-\frac{2 k_{y}^{\prime \prime} k_{y}^{\prime} m_{1}^{2}}{\left(k_{y}^{\prime \prime}+k_{y}^{\prime}\right)^{2}}\right)+\right. \\
& \left.+\frac{8 k_{x} K^{2} m_{1} \operatorname{sh}\left(k_{x} d\right)}{\left(k_{y}^{\prime \prime}+k_{y}^{\prime}\right)}\left(\frac{1+\delta}{\delta^{2}}\right)-\frac{8 K^{2} k_{x}\left(m+m^{\prime}\right) m_{1} \operatorname{sh}\left(2 k_{x} d\right)}{\delta^{2}\left(k_{y}^{\prime \prime}+k_{y}^{\prime}\right)}\right]+ \\
& +\lambda\left[\frac{-\left(m^{2}+m^{\prime 2}\right)}{\delta^{2}}+\frac{2}{\delta^{2}} \frac{k_{y}^{\prime} k_{y}^{\prime \prime} m_{1}^{2}}{\left(k_{y}^{\prime \prime}+k_{y}^{\prime}\right)^{2}}-\frac{2 \operatorname{ch}\left(2 k_{x} d\right)}{\delta^{2}}+\frac{8 K^{2} k_{x} m_{1} \operatorname{sh}\left(k_{x} d\right)}{\delta^{2}\left(k_{y}^{\prime \prime}+k_{y}^{\prime}\right)}\right]+\frac{1}{\delta^{2}}=0 .
\end{aligned}
$$

The features of the interaction between an elastic wave and the moving lattice are determined by the DW velocity. However, expression (24) implicitly contains a dependence on velocity $V_{D}$ owing to the presence of wave vector $k_{y}^{\prime}$ and quantities $m, m^{\prime}$, $m_{1}$, and $\delta$, which are represented in terms of this wave vector. Therefore, this implicit dependence can be demonstrated only numerically, because of the complexity of the resulting expressions.

In order to consider the case of a static lattice $\left(V_{D}=0\right)$, we make the following substitutions in expression (24): $m, m^{\prime} \rightarrow \cos \left(k_{y} \tilde{y}\right), \delta \rightarrow 1, \quad k_{y}^{\prime}=k_{y}^{\prime \prime} \rightarrow k_{y}, \quad$ and $m_{1} \rightarrow 2 \sin \left(k_{y} \tilde{y}\right)$. Then, adding quantities $Q\left(k_{x}, k_{y}\right) \cos ^{2}\left(k_{y} d\right)$ and $Q\left(k_{x}, k_{y}\right) \operatorname{ch}^{2}\left(k_{y} d\right)$, $\left(Q\left(k_{x}, k_{y}\right)=16 K^{2}\left(k_{x} / k_{y}\right) \sin \left(k_{y} d\right) \operatorname{sh}\left(k_{x} d\right)\right)$ to the coefficient of $\lambda^{2}$ in Eq. (24) and subtracting the aforementioned quantities from this coefficient, we obtain after some algebra the dispersion equation for a static lattice:

$$
\lambda^{4}-\left(S_{1}+S_{2}\right) \lambda^{3}+\left(2+S_{1} S_{2}+U\right) \lambda^{2}-\left(S_{1}+S_{2}\right) \lambda+1=0 .
$$

Here, we use the following notation:

$$
\begin{gathered}
S_{1}=2 \cos \left(2 k_{y} d\right)-4 \frac{k_{x}}{k_{y}} K^{2} \sin \left(k_{y} d\right) \operatorname{sh}\left(k_{x} d\right), \\
S_{2}=2 \operatorname{ch}\left(2 k_{x} d\right)-4 \frac{k_{x}}{k_{y}} K^{2} \sin \left(k_{y} d\right) \operatorname{sh}\left(k_{x} d\right), \\
U=16 K^{2} \frac{k_{x}}{k_{y}} \sin \left(k_{y} d\right) \operatorname{sh}\left(k_{x} d\right)\left(\cos \left(k_{y} d\right)-\operatorname{ch}\left(k_{x} d\right)\right)^{2} .
\end{gathered}
$$

A solution to Eq. (25) is a reciprocal fourth-order polynomial [Korn \& Korn, 1968] and can be analytically represented as 


$$
\begin{array}{ll}
\lambda_{1,2}=\frac{y_{1} \pm \sqrt{y_{1}^{2}-4}}{2}, & y_{1}=\frac{\left(S_{1}+S_{2}\right)}{2}+\sqrt{\frac{\left(S_{1}-S_{2}\right)^{2}-4 U}{4}} \\
\lambda_{3,4}=\frac{y_{2} \pm \sqrt{y_{2}^{2}-4}}{2}, & y_{2}=\frac{\left(S_{1}+S_{2}\right)}{2}-\sqrt{\frac{\left(S_{1}-S_{2}\right)^{2}-4 U}{4}} .
\end{array}
$$

When term $U$ in the coefficient of $\lambda^{2}$ equals zero $\left(k_{x}=0\right.$ or $K=0$ and, hence, $S_{1}=2 \cos \left(k_{y} d\right)$ and $S_{2}=2 \operatorname{ch}\left(2 k_{x} d\right)$ ), we obtain the following result from expression (26):

$$
\lambda_{1,2}=\frac{S_{1} \pm \sqrt{S_{1}^{2}-4}}{2}, \quad \lambda_{3,4}=\frac{S_{2} \pm \sqrt{S_{2}^{2}-4}}{2} .
$$

Note that $\lambda_{1,2}$ and $\lambda_{3,4}$ are the roots of the two corresponding quadratic equations. Hence, Eq. (25) can be written as $\left(\lambda^{2}-S_{1} \lambda+1\right)\left(\lambda^{2}-S_{2} \lambda+1\right)=0$ and the solutions can be found with the use of the Vieta theorem and the substitution $\lambda_{1}+\lambda_{1}^{-1}=\cos (2 i \kappa d) \quad(\lambda=\exp (2 i \kappa d)$, $\left.S_{2}=2 \operatorname{ch}\left(2 k_{x} d\right)\right)$. Thus, we obtain

$$
\cos \left(2 \kappa_{1} d\right)=\cos \left(2 k_{y} d\right) . \quad \cos \left(2 \kappa_{2} d\right)=\operatorname{ch}\left(2 k_{x} d\right) .,
$$

For the case under study, this factorization of Eq. (25) corresponds to the extinction of the interaction between acoustic and electric oscillations that is due to the absence of the piezoelectric effect $(K=0)$ or the absence of near-boundary electric oscillations in the domains $\Phi_{j}=0$ [Shuvalov \& Gorkunova,1999] under the conditions for the EAW normal propagation $\left(k_{x}=0\right)$ when the acousto-domain interaction vanishes. A similar result is valid for Eq. (20), which can be represented also as a product of the dispersion relations for independent elastic and electric subsystems.

For the case under study, this factorization of Eq. (25) corresponds to the extinction of the interaction between acoustic and electric oscillations that is due to the absence of the piezoelectric effect $(K=0)$ or the absence of near-boundary electric oscillations in the domains $\Phi_{j}=0$ [Shevyakhov, 1990] under the conditions for the EW normal propagation $\left(k_{x}=0\right)$ when the acousto-domain interaction vanishes. A similar result is valid for Eq. (24), which can be represented also as a product of the dispersion relations for independent elastic and electric subsystems.

When $k_{x}=0$ or $K=0$, the first equation from (28) becomes identity, a result that means that a purely elastic wave ${ }^{1}$ propagates in the crystal along the normal to the domain planes with propagation constant $k_{y}$ (i.e., a bulk shear wave is excited in an infinite crystal). It follows from the second identity that the Bloch number is either purely imaginary $(K=0)$, i.e., a solution does not exist, owing to the formation of a continuous forbidden band) or $\left(k_{x}=0\right)$ equal to $2 \pi n /(2 d)$, where $n$ is integer. In the second case $\left(k_{x}=0\right)$, the boundary conditions are satisfied if $A_{1}=0, B_{1}=0, A_{2}=0, B_{2}=0$, and $B_{2}=0$ (i.e., all of the fields are identically zero). Therefore, the solution is degenerate and must be disregarded. Excluding both variants $\left(k_{x}=0\right.$ and $\left.K=0\right)$ as the variants that do not allow the acousto-domain interaction, we assume that Eq. (24) always describes coupled electroacoustic oscillations for a static superlattice.

\footnotetext{
1 At $K \neq 0$, this wave is accompanied by the in-phase oscillations of the electric field (see the first term in expression (14)).
} 


\subsection{Solution and analysis of the dispersion equation}

A numerical solution to Eq. (24) that has been obtained with the use of expression (27) is shown in Fig. 3. In this study, the calculations are performed for a barium titanate crystal $\left(\left(\mathrm{BaTiO}_{3}\right)\right.$ with the following parameters: the crystal density $\rho=5 \mathrm{~g} / \mathrm{cm}^{3}, K^{2} \approx 0.37$, the velocity of the transverse waves in the absence of the polarizing field $c_{t 0}=\left(c_{44} / \rho\right)^{1 / 2}=2 \times 10^{5}$ $\mathrm{cm} / \mathrm{s}, \varepsilon_{1}=5 \times 10^{3}$, and $\mathrm{e}_{15} \approx 3 \cdot 10^{6} \mathrm{~g} /(\mathrm{cm} \mathrm{s})$. Figures $3 \mathrm{a}$ and $3 \mathrm{~b}$ respectively demonstrate the real and imaginary parts of the roots of the dispersion equation for the static (dashed lines) and moving (solid lines) superlattices. The results are obtained for the propagation angle $\theta^{\prime \prime}=\pi / 3$ and the positive direction of DW motion $\left(V_{D}>0\right)$. The numbers of the curves correspond to the root numbers $\left(\lambda_{1}, \lambda_{2}, \lambda_{3}, \lambda_{4}\right)$. The roots $\lambda_{1,2}=\exp \left(2 i \kappa_{1,2} d\right)$ in expression (27) (Fig. 3a, curves 1,2) are purely real. This means that the Bloch wave numbers $\kappa_{1,2}$ are purely imaginary for any wave number $k$ and correspond to the modes that are forbidden for the given periodic structure. Note that the shapes of curves 1 and 2 remain almost unchanged when the motion is taken into account. The roots $\lambda_{3,4}=\exp \left(2 i_{\kappa_{3,4}} d\right)$ (Fig. 3a, curves 3,4) describe propagating waves. Below, we consider only the spectral properties of the propagating eigenmodes of the superlattice.
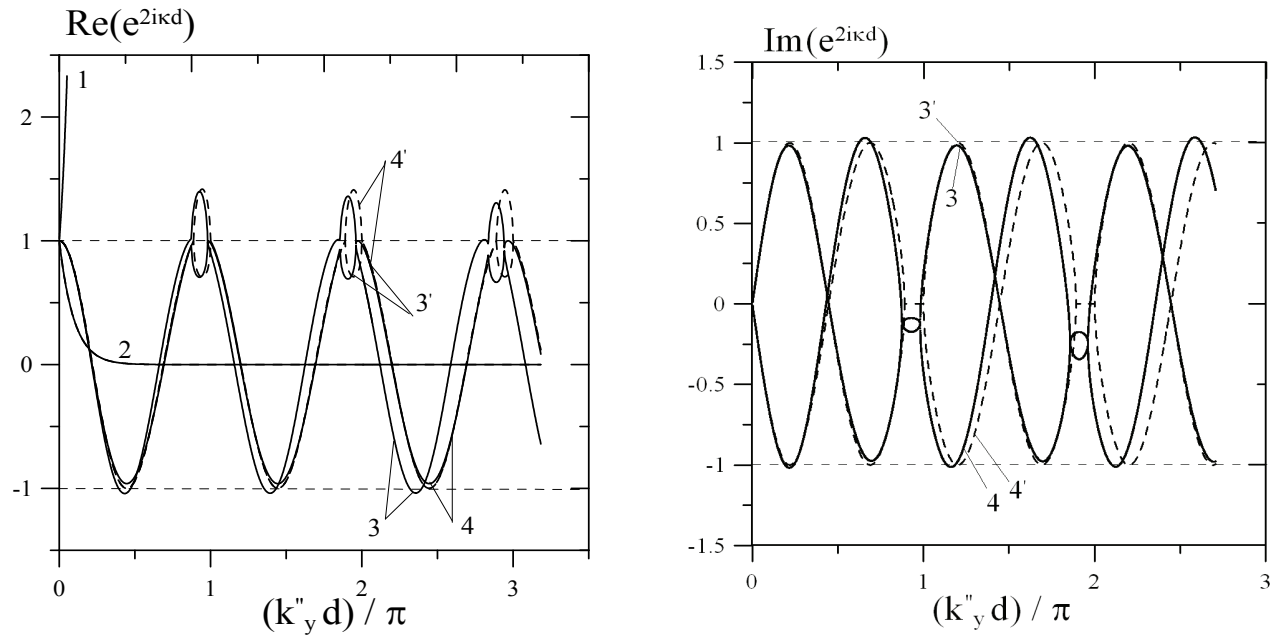

Fig. 3. (a) Real parts of the roots $\lambda_{1}, \lambda_{2}, \lambda_{3}\left(3,3^{\prime}\right)$, and $\lambda_{4}\left(4,4^{\prime}\right)$ and plotted as a function of $\left(k_{y}^{\prime \prime} \mathrm{d}\right) / \pi$ for various values of $\mathrm{V}_{\mathrm{D}}:\left(1,2,3^{\prime}, 4^{\prime}\right) 0$ and $(3,4) 0.01 \mathrm{v}$. Curves $3^{\prime}$ and $4^{\prime}$ coincide except in the region of the loop-shaped segments, (b) Imaginary parts of the roots $\lambda_{3}\left(3,3^{\prime}\right)$, and $\lambda_{4}\left(4,4^{\prime}\right)$ plotted as a function of $\left(k_{y}^{\prime \prime} \mathrm{d}\right) / \pi$ for various values of $\mathrm{V}_{\mathrm{D}}:(3,4) 0.01 \mathrm{v}, 3^{\prime}, 4^{\prime}$

It is seen from Fig. 3a that, in the static case, the real parts of roots 3 and 4 are cosines whose arguments contain the Bloch number. The imaginary parts of these roots are the sines of the above argument. It follows from the comparison of Figs. $3 \mathrm{a}$ and $3 \mathrm{~b}$ that curves 3 and 4 describe counterpropagating waves. Indeed, the spectral characteristics of the modes of the static superlattice that propagate in the opposite directions must differ only by the sign of the Bloch wave number: The positive and negative Bloch wave numbers respectively correspond to the waves that propagate in the positive and negative direction of the $\tilde{y}$ axis. 
The calculations show that an increase in propagation angle $\theta^{\prime \prime}$ leads to an increase in the width of the loop fragment (Fig. 3) on the curve of the real part of the root. In accordance with Fig. 3b, the imaginary part of the root is absent for this spectral interval in the case of the dashed curves. Thus, the Bloch wave number, which is the propagation constant averaged over the lattice period, is purely imaginary and the wave propagation is impossible, so that the corresponding fragment is a forbidden band [Balakirev \& Gilinskii, 1982].

The appearance of forbidden bands is obvious. In accordance with Fig. 3a, the Bloch vector exhibits variations in the transmission band, so that an integer number of half eigenwaves is realized at the band edges. At each boundary, this circumstance corresponds to the in phase summation of the forward wave and the wave that is reflected from the edge whose distance from the boundary equals the period of the structure. In contrast, the forward and reflected (antiphase) waves are mutually cancelled in the band gaps.

An increase in angle $\theta^{\prime \prime}$ results in a simultaneous increase in the widths of allowed and forbidden bands. However, their number decreases in the range of wave vectors $0<k^{\prime \prime}<2$ $\times 10^{5} \mathrm{~cm}^{-1}$ (the frequency range $0<\omega^{\prime \prime}<5 \times 10^{10} \mathrm{~s}^{-1}$ ), which is common to all of the plots. For the given domain size $d=10^{-4} \mathrm{~cm}$, dimensionless parameter $k d$ ranges from 0 to 20 . Thus, the calculations are performed almost in the absence of limitations on this parameter.

In addition, it is seen from Fig. 3 that the DW motion causes the Doppler shift of eigenwaves, which eliminates the degeneration of the roots of the dispersion relation. This fact is manifested in the graphs: The real parts of roots $\lambda_{3}$, and $\lambda_{4}$ for the counterpropagating EWs are not equal and are described by different curves. Curve 4 corresponds to the wave that propagates in the direction opposite to the $\tilde{y}$ axis, and curve 3 corresponds to the wave that propagates along the $\tilde{y}$ axis. In accordance with Fig. 2, at $V_{D}>0$, , the forbidden bands exhibit a shift to the long-wavelength region. The comparison with the calculated results obtained for $V_{D}<0$ shows that, relative to the forbidden band at $V_{D}=0$, the forbidden bands are symmetrically shifted to the shortwavelength region.

For relatively large $\left(\kappa_{3,4} d\right) / \pi$, we observe a larger difference between curves 3 and 4 . This means that, as the number of the oscillation mode increases, the effect of the DW motion on the EW spectrum strengthens. This result is in agreement with the results from [Vilkov, 2007], where the spectral properties of magnetostatic waves are analyzed with allowance for the motion of a DW superlattice. In the spectral fragments with $0 V_{D} \neq 0$ (Fig. 3) that coincide with the spectral forbidden band of the static superlattice, a shifted forbidden band is formed. Thus, the amplitudes of eigenwaves in this band of the moving superlattice contain oscillating factors. In addition, new forbidden bands emerge at $V_{D} \neq 0$ in the spectral fragments where the real and imaginary parts of the roots of the dispersion equation are greater than unity. This circumstance is due to the fact that the lattice motion results in an additional phase shift between the counterpropagating waves.

The calculated results show that, if the Bloch wave number in the first spectral band in Fig. 3 is approximately equal to $k_{y}^{\prime \prime}$ at small angles $\theta^{\prime \prime}$, a significant difference between $\kappa$ and $k_{y}^{\prime \prime}$ can be realized at large angles. For example, at $\theta=80^{\circ}$, the propagation constant $\kappa$ averaged over the superlattice period is approximately two times greater than $k_{y}^{\prime \prime}$, a result that indicates a significant effect of the superlattice on the spectrum of bulk EWs. The lattice motion leads to a difference between the Bloch wave numbers of the counter propagating waves and, hence, to differences between the EW propagation velocities and between the field profiles that characterize EWs. Thus, the mutual nonreciprocity of the EW propagation induced by the lattice motion needs further analysis. 


\subsection{Calculation of the EW displacement profiles and phase}

To calculate the EAW displacement profiles, we employ the periodicity condition from [Bass et al, 1989]: On the boundaries with the coordinates $\tilde{y}=0$ and $\tilde{y}=2 d$, the fields can differ only by the phase factor (the Floquet theorem); i.e.,

$$
u(0)=u(2 d) \exp (2 i \kappa d), \quad \varphi(0)=\varphi(2 d) \exp (2 i \kappa d), \quad \Phi(0)=\Phi(2 d) \exp (2 i \kappa d),
$$

Substituting expressions for the fields (16) and (17) with allowance for formulas (25) into boundary conditions (20) on two boundaries of an elementary cell $(\tilde{y}=0$ and $\tilde{y}=d)$, we obtain a system of eight homogeneous algebraic equations for amplitudes $A_{1,2}, B_{1,2}, C_{1,2}$ and $\mathrm{D}_{1,2}$.
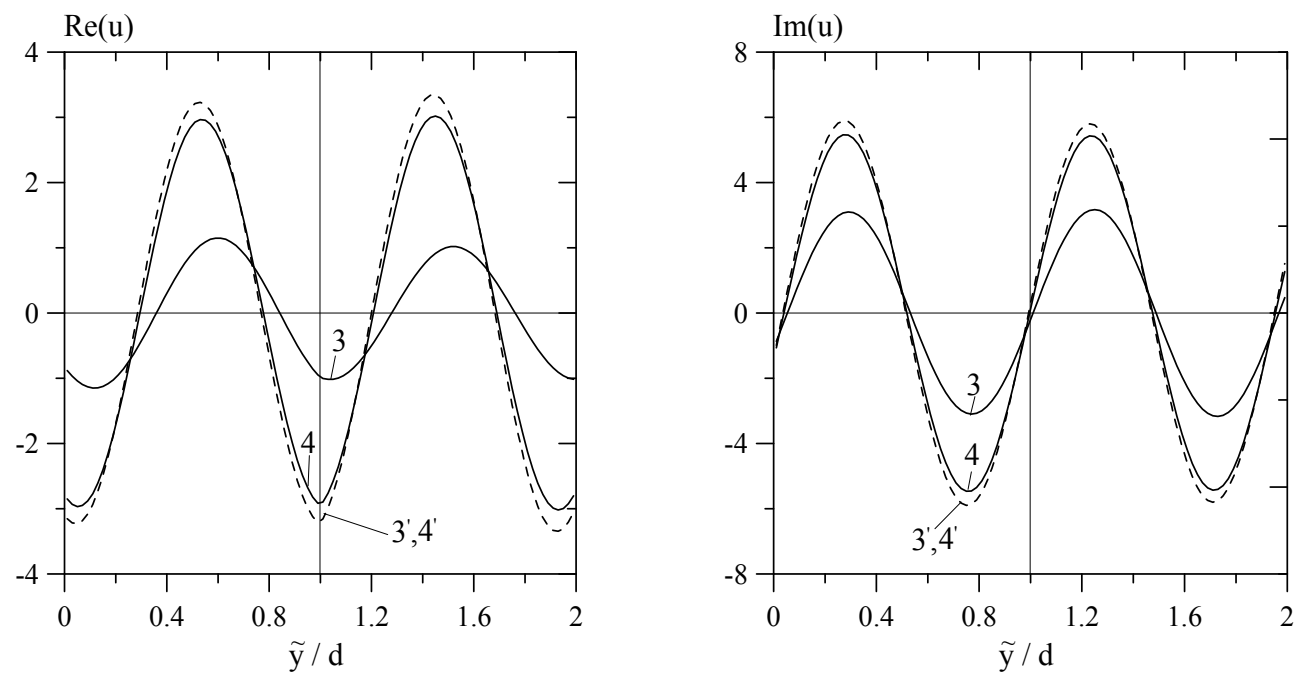

Fig. 4. (a) Real imaginary parts of the amplitude profile of the EW shear displacement for the wave $\mathrm{k}$ vector $=131300 \mathrm{~cm}-1$ corresponding to the allowed band, $\theta^{\prime \prime}=60^{\circ}$, and $\mathrm{V}_{\mathrm{D}}=$ (dashed lines) 0 and $(3,4) 0.01 \mathrm{v}$, (b) Imaginary parts of the amplitude profile of the EW shear displacement for the wave $\mathrm{k}$ vector $=131300 \mathrm{~cm}-1$ corresponding to the allowed band, $\theta^{\prime \prime}=60^{\circ}$, and $V_{\mathrm{D}}=$ (dashed lines) 0 and $(3,4) 0.01 \mathrm{v}$

Assuming that one of the amplitudes (e.g., $A_{1}$ ) equals unity, we solve seven of eight equations and represent all of the amplitudes in terms of the selected one. Then, we substitute the resulting expressions into formulas (16) and (17) and, with allowance for the solutions to Eq. (25), find the desired field profiles within the lattice period $0<\tilde{y}<2 d$.

Figure 3 demonstrates the real and imaginary parts of the shear wave displacement in the crystal with the static (dashed curves) and moving (solid curves) superlattices for the positive motion of the lattice $\left(V_{D}>0\right)$. Figure 4 corresponds to the wave vector falling in the EW spectral band in Fig. 3. It is seen that the real and imaginary parts of the displacement respectively correspond to the symmetric and antisymmetric modes. Note that the presented set of mode profiles is universal for given $k^{\prime \prime}$ and the sum of the lattice spatial harmonics, 


$$
u(\tilde{y})=\sum_{n=-\infty}^{\infty} u_{n} \exp \left[i\left(\kappa+\frac{2 \pi n}{d}\right) \tilde{y}\right] .
$$

This circumstance is obvious because, in accordance with expression (30), physically nonequivalent states in the periodic structure correspond only to the range $-\pi / d<\kappa<\pi / d$. It is seen from Fig. 4 that the amplitudes of counterpropagating waves coincide for the static lattice and significantly differ for the moving lattice. In particular, the amplitude of the wave propagating along the axis (curve 3 ) is approximately two times smaller than the amplitude of the wave propagating in the opposite direction (curve 4). This amplitude imbalance of the counterpropagating waves added to the misphasing can be interpreted via a variation produced in the wave energy owing to an external source that provides for the DW motion. The mechanism that controls the amplitude variations involves the Doppler frequency shifts: The wave with the maximum Doppler shift (Fig. 4, curve 3) exhibits the largest amplitude difference relative to the wave of the static superlattice (Fig. 4, dashed line). In contrast, note minor variations in the amplitude of the wave (Fig. 4, curve 4) whose spectral characteristics other than kare initially identical to the spectral characteristics of the wave in the static lattice.

Each of the harmonics in expression (30) is characterized by the same displacement profile, whereas the phase velocities are different [Balakirev \& Gilinskii, 1982]:

$$
\begin{aligned}
& \left|\vec{v}_{f n}^{\prime \prime}\right|=\omega^{\prime \prime}\left[\left(\kappa_{4}+2 \pi n / d\right)^{2}+k_{x}^{2}\right]^{-1 / 2} \\
& \left|\vec{v}_{f n}^{\prime}\right|=\omega^{\prime}\left[\left(\kappa_{3}+2 \pi n / d\right)^{2}+k_{x}^{2}\right]^{-1 / 2}
\end{aligned}
$$

Here, $\left|\vec{v}_{f n}^{\prime}\right|$ and $\left|\vec{v}_{f n}^{\prime \prime}\right|$ are the magnitudes of the phase velocities of the waves that propagate in the direction of the PDS motion and in the opposite direction. For the static

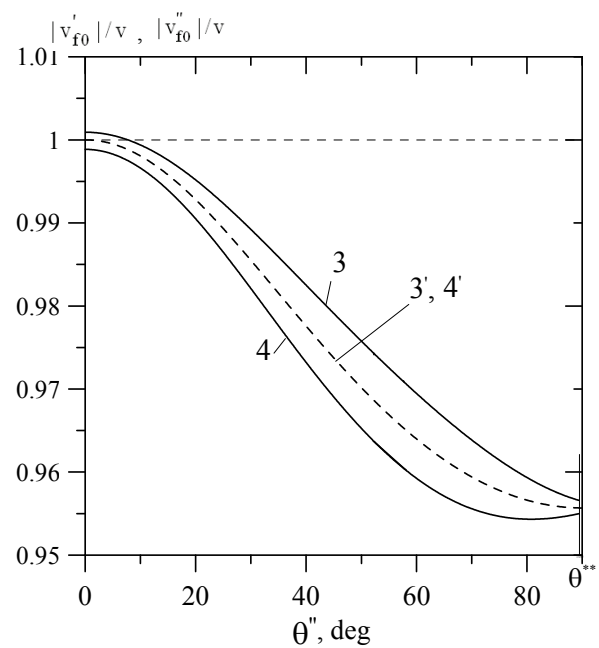

Fig. 5. Plots of the EW phase velocities vs. the propagation angle $\theta^{\prime \prime}$ for $V_{\mathrm{D}}=$ (dashed line) 0 and $(3,4) 0.01 v$. Curves 3 and 4 correspond to the EWs propagating along and opposite to the $y$ axis, respectively 
lattice, we have $\left|\vec{v}_{f n}^{\prime \prime}\right|=\left|\vec{v}_{f n}^{\prime}\right|$ It follows from expression (31) that, for the $n$th harmonic, the phase velocity can be infinitesimal. For certainty, we set the phase velocity of the Bloch wave in the following way: $\left|\vec{v}_{f}^{\prime \prime}\right|=\left|\vec{v}_{f 0}^{\prime}\right|$, and $\left|\vec{v}_{f}^{\prime}\right|=\left|\vec{v}_{f 0}^{\prime}\right|$. The dependences of the phase velocities of the Bloch waves on propagation angle $\theta^{\prime \prime}$ calculated for $V_{D}>0$ are presented in Fig. 5.

We do not consider the limiting angles $\theta^{\prime \prime}=0^{\circ}$, and $\theta^{\prime \prime}=90^{\circ}$ and the nearest vicinities for the following reasons. In the first case $\left(\theta^{\prime \prime}=0^{\circ}\right)$, the electric-field retardation must be taken into account in the correct analysis of the acousto-domain interaction [Balakirev \& Gilinskii, 1982]. For a moving PDS, the transition $\theta^{\prime \prime} \rightarrow 90^{\circ}$ is impossible because of the limitation $\theta^{\prime}<\theta^{* *}$ related to the termination of the acousto-domain interaction. It is seen from Fig. 5 that the greatest difference (about $2 \%$ ) between phase velocities $\left|\vec{v}_{f n}^{\prime}\right|$ and $\left|\vec{v}_{f n}^{\prime \prime}\right|$ is realized in the interval $60^{\circ}<\theta<70^{\circ}$ and can be experimentally detected. The phase-velocity nonreciprocity can be caused by the Doppler separation of the frequencies of counterpropagating waves and a simultaneous variation in the Bloch wave numbers that is due to different spatial-temporal periodicities in two opposite directions induced by the uniformly moving DW superlattice.

\section{Reflection of electroacoustic waves from a system of moving domain walls in a ferroelectric}

\subsection{The statement of the problem}

In the previous section we have shown, that significant modification of the spectrum of modes of shear waves are possible owing to motion of boundaries of a superlattice of a ferroelectric. It can be assumed, that motion of domain boundaries will exert the strong influence on reflection of electroacoustic waves from a lattice of domain boundaries. The important controlling role of the velocity of domain-wall motion is indirectly confirmed by the results obtained in [Shevyakhov, 1990], which indicate that the interaction of a bulk electroacoustic wave with a single moving domain wall in the case of a noticeable change in the amplitude coefficients (the range where the angles of incidence are not very small) is accompanied by the Doppler frequency transformation. By analogy with the static case [Shuvalov \& Gorkunova, 1999], it can be expected that, for a system of moving domain walls, the reflection can be considerably enhanced in the direction of Bragg angles. At the same time, the velocity of domain-wall motion will serves as a new parameter that is convenient for controlling the reflection and transmission of waves in combination with their frequency shifts. In this section the interaction of electroacoustic waves with a periodic domain structure formed in a tetragonal ferroelectric by a finite number of uniformly moving 180-degree domain walls is considered in the quasi-static approximation.

The schematic diagram of the problem is depicted in Fig. 6. We consider the same the ferroelectric and the same the periodic structure, as in the previous section, but at that periodic structure is formed by finite number 180-degree domain boundaries. It is assumed that, in the $y$ direction, the domain-wall lattice, which consists of $2 N$ domains for the structure "+-" (Fig. 6a) or 2N+1 domains for the structure "++" (Fig. 6b), has a period 2d, so that $d>>\Delta$ (where $d$ is the distance between neighboring domain walls and $\Delta$ is the domain-wall thickness). On both sides, the lattice uniformly moving at the velocity $V_{D}$ $\|y\|[010]$ is surrounded by semi-infinite single-domain crystal regions (the external numbers (with respect to the lattice) of domains are $n=0$ and $2 N+1$ for the structure "+-" and $n=0$ and $2 N+2$ for the structure " $++")$. In order to avoid a significant structural 
sensitivity of domain walls to the motion regime, we will restrict our consideration to the velocities, we will restrict our consideration to the velocity of shear waves in a singledomain sample [Sosnin. \& Strukov 1970; Vainshtein,1988]. Under the above conditions, the motion of domain walls can be considered to be specified $\left(V_{D}=\right.$ const $)$ with the current coordinates $y_{m}=V_{D} t+m d$, where $t$ is the time and $m=0, \pm 1, \pm 2, \ldots$. Correspondingly, the domain walls are assumed to be geometrically thin and structureless $(k \Delta<<1$, where $k$ is the wave number of the electroacoustic wave).

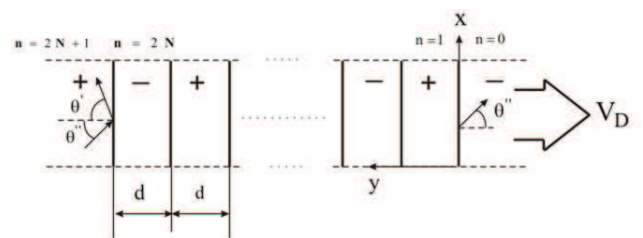

(a)

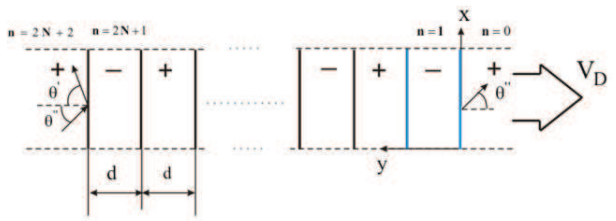

(b)

Fig. 6. (a) Schematic diagram of the problem: a moving domain lattice is surrounded by semi-infinite single-domain crystal regions with (a) opposite (structure "+-")directions of polarization. Tilted vectors indicate the direction of propagation of the electroacoustic wave. The arrow indicates the direction of the lattice motion, (b) Schematic diagram of the problem: a moving domain lattice is surrounded by semi-infinite single-domain crystal regions with identical (structure "++") directions of polarization. Tilted vectors indicate the direction of propagation of the electroacoustic wave. The arrow indicates the direction of the lattice motion

We assume, that $j=1$ and 2 stand for domains of the "+" and "-" types with positive and negative piezoelectric moduli, respectively according $\mathrm{Eq}(9)$. Other differences between domains are absent. We also assume that the domain with the number $n=0$ is a domain of the "-" type for the structure "+-" and a domain of the "+" type for the structure "++" and that the domain with the number $n=2 N+1$ or $n=2 N+2$ is a domain of the "+" type (Fig. 6). Moreover, it also is assumed that the electroacoustic wave with the wave $\mathbf{k}^{\prime \prime}=\left(k^{\prime \prime} \sin \theta^{\prime \prime}, k^{\prime \prime} \cos \theta^{\prime \prime}, 0\right)$, propagates in the $x 0 y$ plane and is incident on the domain lattice at the angle $\theta^{\prime \prime}\left(\theta^{\prime \prime} \neq 0\right)$. In view of the expected Doppler frequency shift [Shevyakhov, 1990], another wave vector $\mathbf{k}^{\prime}=\left(k^{\prime} \sin \theta^{\prime}, k^{\prime} \cos \theta^{\prime}, 0\right)$ is assigned to reflected waves (Fig. 6). The initial equations remain without changes and to be defined by the equations (9), (13), (14). As a result of the phase conjugation of the fields at moving domain walls of the lattice, the wave characteristics of the reflected and transmitted electroacoustic waves are related by the expressions (15).

In order to avoid the analysis of the additional refraction scheme when the reflected wave becomes adjusting with respect to the incident wave, it is necessary to introduce the constraint on the angle of incidence $\theta^{\prime \prime}<\theta^{*}$ [Shevyakhov, 1990], where

$$
\theta^{*}=\arccos \left[-2\left(V_{D} / v\right) /\left(1+\left(V_{D} / v\right)^{2}\right)\right]
$$

\subsection{Technique for calculating the transmittance and reflectance of electroacoustic waves}

In the domain with the number $\mathrm{n}=2 \mathrm{~N}+1$ for the structure "+-" or with the number $\mathrm{n}=2 \mathrm{~N}$ +2 for the structure "++," we have the incident and reflected electroacoustic waves and the 
electric field wave localized at the boundary $\tilde{y}=2 \mathrm{Nd}$ or $\tilde{y}=2 \mathrm{Nd}+\mathrm{d}$ (Fig. 6). The shear displacements and the potentials of these waves as the solutions to Eqs. (13) can be written in the form

$$
\begin{gathered}
u_{2 N+1,2 N+2}(\tilde{y})=\left(\exp \left(i k_{y}^{\prime \prime}(\tilde{y}-2 N d)+R \exp \left(-i k_{y}^{\prime}(\tilde{y}-2 N d)\right)\right) \exp \left(i\left(k_{x} \tilde{x}-\Omega \tilde{t}\right)\right)\right. \\
\Phi_{2 N+1,2 N+2}(\tilde{y})=\left(C \exp \left(-k_{x}(\tilde{y}-2 N d)\right) \exp \left(i\left(k_{x} \tilde{x}-\Omega \tilde{t}\right)\right)\right.
\end{gathered},
$$

where the amplitude of the incident wave is taken to be unity, $R$ is the reflectance, and $C$ is the amplitude of the electric wave potential. Correspondingly, for the domain with the number $n=0$ for both structures, we obtain

$$
\begin{aligned}
& u_{0}(\tilde{y})=W \exp \left(i k_{y}^{\prime \prime} \tilde{y}\right) \exp \left(i\left(k_{x} \tilde{x}-\Omega \tilde{t}\right)\right) \\
& \Phi_{0}(\tilde{y})=D \exp \left(k_{x} \tilde{y}\right) \exp \left(i\left(k_{x} \tilde{x}-\Omega \tilde{t}\right)\right)
\end{aligned} .
$$

Here, $W$ is the transmittance of the electroacoustic wave and $D$ is the amplitude of the localized electric field wave. The solutions to Eqs. (13) in the region within the lattice for a domain of the "+" type are sought in the following form:

$$
\begin{aligned}
& u_{1}(\tilde{y})=\left(A_{1} \exp \left(i k_{y}^{\prime \prime} \tilde{y}\right)+A_{2} \exp \left(-i k_{y}^{\prime} \tilde{y}\right)\right) \exp \left(i\left(k_{x} \tilde{x}-\Omega \tilde{t}\right)\right) \\
& \Phi_{1}(\tilde{y})=\left(C_{1} \exp \left(k_{x} \tilde{y}\right)+C_{2} \exp \left(-k_{x} \tilde{y}\right)\right) \exp \left(i\left(k_{x} \tilde{x}-\Omega \tilde{t}\right)\right)^{\prime}
\end{aligned}
$$

In the region of a domain of the "-" type, the solution is represented in a similar way

$$
\begin{aligned}
& u_{2}(\tilde{y})=\left(B_{1} \exp \left(i k_{y}^{\prime \prime} \tilde{y}\right)+B_{2} \exp \left(-i k_{y}^{\prime} \tilde{y}\right)\right) \exp \left(i\left(k_{x} \tilde{x}-\Omega \tilde{t}\right)\right) \\
& \Phi_{2}(\tilde{y})=\left(D_{1} \exp \left(k_{x} \tilde{y}\right)+D_{2} \exp \left(-k_{x} \tilde{y}\right)\right) \exp \left(i\left(k_{x} \tilde{x}-\Omega \tilde{t}\right)\right)^{\prime}
\end{aligned}
$$

In order to match the fields of the domains in the rest system at the domain walls, in the quasi-static nonrelativistic approximation, we use the standard requirements for the continuity of the shear displacements, the potentials, the shear components of the stress tensor, and the electric induction components $y$ (see Eqs(20)). At the same time, The matrices $\mathbf{M}_{1}=\mathbf{M}_{1}(d)$ and $\mathbf{M}_{2}=\mathbf{M}_{2}(d)$ (see Eqs.(23)) relate the fields at the initial and final points of the same domain layer. Their product determines the transition matrix at the period of the structure $\mathbf{M}=\mathbf{M}_{1} \mathbf{M}_{2}$.

Let us assume that, in expressions (20) and (22), the quantities determining the entire set of waves in the domain are designated as $u_{0}, \alpha_{0}, \varphi_{0}$, and $\sigma_{0}$ in the domain with the number $n=$ 0 . Then, their relation to the quantities $u_{2 N}, \alpha_{2 N}, \varphi_{2 N}$, and $\sigma_{2 N}$ in the domain with the number $n=2 N$ in the structure "+-" can be found using the 4-by-4 transformation matrix $\mathbf{M}$ at the period of the structure as follows:

$$
\left(\begin{array}{l}
u_{0} \\
\alpha_{0} \\
\varphi_{0} \\
\sigma_{0}
\end{array}\right)=\mathbf{M}^{N}\left(\begin{array}{l}
u_{2 N} \\
\alpha_{2 N} \\
\varphi_{2 N} \\
\sigma_{2 N}
\end{array}\right)
$$

where $N$ is the exponent of the matrix $\mathbf{M}$ and, simultaneously, the number of "whole" unit cells formed by domains of the "-" and "+" type in the lattice. For the structure "++," the 
relation of the fields of the domain with the number $n=0$ to the fields of the last domain in the lattice will be somewhat different as a result of the additional transformation of the waves by the last domain; that is,

$$
\left(\begin{array}{l}
u_{0} \\
\alpha_{0} \\
\varphi_{0} \\
\sigma_{0}
\end{array}\right)=\mathbf{M}^{N} \mathbf{M}_{2}\left(\begin{array}{l}
u_{2 N+1} \\
\alpha_{2 N+1} \\
\varphi_{2 N+1} \\
\sigma_{2 N+1}
\end{array}\right)
$$

The reflectance for the structure "+ -" (structure "++") can be determined from the boundary conditions at the domain wall between the domain with the number $n=2 \mathrm{~N}$ ( $n=$ $2 N+1)$ and the domain with the number $n=2 N+1(n=2 N+2)$, i.e., by equating the corresponding wave parameters at $\tilde{y}=2 N d(\tilde{y}=2 N d+d)$. Then, we multiply the obtained identities by $\mathbf{M}^{\mathrm{N}}\left(\mathbf{M}^{\mathrm{N}} \mathbf{M}_{2}\right)$ from the left and right and, with the use of relationships (20)-(22), (33), (34), (37), and (38), find the systems of four equations for determining the reflectance and transmittance: for the structure "+-,"

$$
\left(\begin{array}{c}
W \\
-\left(i c_{44}^{*} k_{y}^{\prime \prime}\right) W-e_{15} k_{x} D \\
-\left(4 \pi e_{15} / \varepsilon_{1}\right) W+D \\
D k_{x}
\end{array}\right)=\mathbf{M}^{\mathrm{N}}\left(\begin{array}{c}
1+R \\
-\left(i c_{44}^{*} k_{y}^{\prime \prime}\right)+\left(i c_{44}^{*} k_{y}^{\prime}\right) R-e_{15} k_{x} C \\
\left(4 \pi e_{15} / \varepsilon_{1}\right)(1+R)+C \\
-C k_{x}
\end{array}\right)
$$

and for the structure ",++ ,

$$
\left(\begin{array}{c}
W \\
-\left(i c_{44}^{*} k_{y}^{\prime \prime}\right) W+e_{15} k_{x} D \\
\left(4 \pi e_{15} / \varepsilon_{1}\right) W+D \\
D k_{x}
\end{array}\right)=\mathbf{M}^{\mathrm{N}} \mathbf{M}_{2}\left(\begin{array}{c}
1+R \\
-\left(i c_{44}^{*} k_{y}^{\prime \prime}\right)+\left(i c_{44}^{*} k_{y}^{\prime}\right) R-e_{15} k_{x} C \\
\left(4 \pi e_{15} / \varepsilon_{1}\right)(1+R)+C \\
-C k_{x}
\end{array}\right)
$$

Here, $D, C, R$, and $W$ are unknown quantities. The expressions obtained for the reflectance and transmittance from relationships (39) and (40) are cumbersome and difficult for the analysis. In this respect, further calculations of the reflectance were performed numerically. As follows from [Shuvalov \&. Gorkunova, 1999], the analytical formulas for the reflectance of electroacoustic waves from the static lattice can be obtained in a simple form only within the short-wavelength approximation. The magnitude of the reflectance in this approximation has the form [Shuvalov \&. Gorkunova, 1999]

$$
|R|^{2}=\frac{q_{\theta}^{4}}{q_{\theta}^{4}+\sin ^{2}(\kappa d) / \sin ^{2}(2(N+1) \kappa d)}
$$

where $\cos (2 \kappa d)=2\left[\cos \left(k_{y} d\right)-q_{\theta}^{2} \sin \left(k_{y} d\right)\right]^{2}-1, q_{\theta}^{2}=K^{2} \operatorname{tg}\left(\theta^{\prime \prime}\right)$, and $\kappa$ is the Bloch wave number.

\subsection{Calculation of the reflectance of electroacoustic waves from the moving lattice}

Before proceeding to the analysis of the influence of the domain-wall motion on the reflectance spectrum of electroacoustic waves, it should be noted that the results of the 
numerical calculations of the reflectance from the system of equations (40) in the case of the static lattice are in complete agreement with those obtained in [Shuvalov \&. Gorkunova, 1999]. In particular, in the short-wavelength range, the value of $|R|$ determined from the approximate formula (41) agrees well with the result of the exact numerical calculations. Since the number of peaks (the main peak plus secondary peaks) in one band gap is equal to the number of domains in the lattice, in our work, the modification of the spectrum due to the domain-wall motion was demonstrated using small numbers $N$ in order to provide the clearness of the results.

The dependences of the magnitude of the reflectance of electroacoustic waves on the reduced normal component of the wave vector at the fixed angle of incidence $\left(\theta^{\prime \prime}=30^{\circ}\right)$ for the structures "++" and "+-" are plotted in Figs. 7 and 8, respectively. The calculated data presented in these and subsequent figures were obtained for the equidistant lattice of domains with $d=10^{-4} \mathrm{~cm}$ in the barium titanate crystal $\mathrm{BaTiO}_{3}$ with the following parameters: the density of the crystal is $\rho=5 \mathrm{gr} / \mathrm{sm}^{3}, \mathrm{~K}^{2} \approx 0.37$, the velocity of transverse waves in the absence of piezoelectric effect is $v=\left(c_{44} / \rho\right)^{1 / 2}=2 \cdot 10^{5} \mathrm{~cm} / \mathrm{s}$. In Figs. 7 and 8 , the dashed lines show the dependences $|R|\left(2 k_{y} \| d / \pi\right)$ for the static lattice according to the calculations from the system of equations (40). The thick lines in Figs. 7 and 8 depict the dependences $|R|\left(2 k_{y}^{\prime \prime} d / \pi\right)$ for the lattice moving away (the direction of domain-wall motion is opposite to the direction of the $Y$ axis, $\left.V_{D}<0\right)$. It can be seen that the domain-wall motion noticeably modifies the reflectance spectrum of electroacoustic waves: all peaks in the spectrum are broadened, increase in the intensity, and are shifted toward the shortwavelength range. In this case, the larger the ratio $\left(2 k_{y}^{\prime \prime} d / \pi\right)$, the larger the shift, so that the maximum of the magnitude of the reflectance can give way to its minimum.

It can be seen from the behavior of the thin lines in Figs. 7 and 8 that, in the case of the approaching lattice (the direction of domain wall motion coincides with the direction of the $Y$ axis, $\left.V_{D}>0\right)$, the changes in the spectrum are as follows: the peaks in the reflectance spectrum are narrowed, decrease in the intensity, and are shifted toward the longwavelength range. In this case, the larger the ratio $\left(2 k_{y}^{\prime \prime} d / \pi\right)$, the larger the shift. Furthermore, it was revealed that the higher the velocity $V_{D}$, the stronger the manifestation of the above changes in the spectrum. This effect of the shift in the spectra for the moving lattice $\left(V_{D}<0, V_{D}>0\right)$ with respect to the spectrum of the static lattice is explained by the Doppler shift in the frequency of the electroacoustic wave due to its interaction with the moving domain walls and, in actual fact, represents an analog of Mandelstam -Brillouin scattering [Fabelinskii, 1968].It can be seen from Figs. 3,8 and Fig.3a that, when the wave number corresponds to the band gap of the Bloch spectrum, the magnitude of the reflectance reaches a maximum; i.e., there appears a Bragg peak. The condition for the appearance of this peak is a correlated reflection of electroacoustic waves from all domain walls in the lattice.

A comparison of the reflectance spectra of electroacoustic waves for the structures "++" (Fig. 7) and "+-" (Fig. 8) reveals several main differences. The first difference between the two reflectance spectra manifests itself in the range of the wave number $k=0$, i.e., for an infinite wavelength. At $k \rightarrow 0$, the reflectance tends to zero for the structure " ++ " and to the reflectance for a single domain wall for the structure "+-" [Shevyakhov, 1990]. Physically, this difference in the behavior of the spectra can be explained as follows. The shear wave 


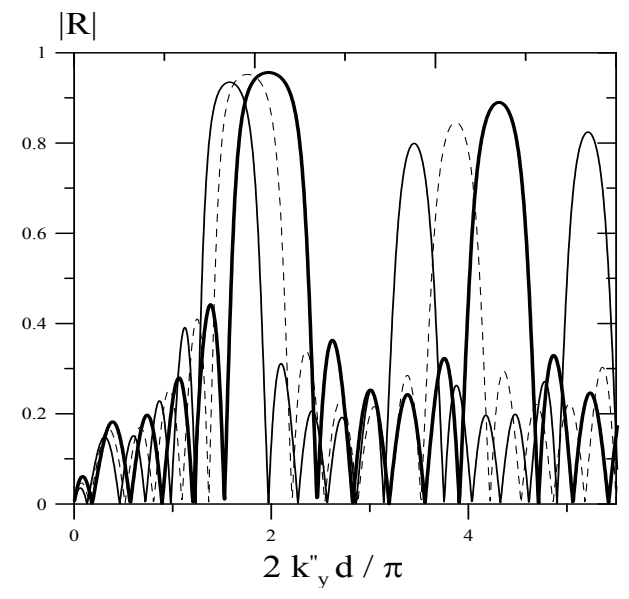

Fig. 7. Dependences of the magnitude of the reflectance $|R|$ on the quantity $\left(k_{y}^{\prime \prime} d\right) / \pi$ for the structure " ++ " consisting of five domains $(N=2)$ at the velocities $V_{D}=($ dashed line $) 0$, (thin line) $0.1 v$, and (thick line) $-0.1 v$

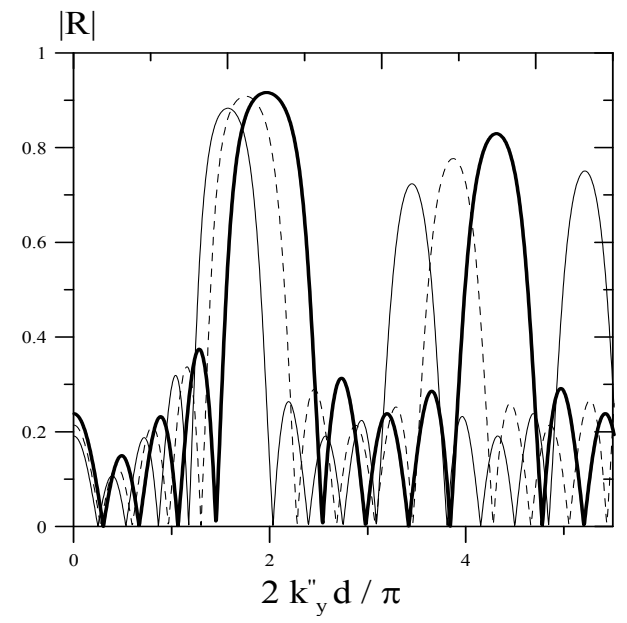

Fig. 8. Dependences of the magnitude of the reflectance $|R|$ on the quantity $\left(k_{y}^{\prime \prime} d\right) / \pi$ for the structure " $+{ }^{\prime}$ " consisting of four domains $(N=2)$ at the velocities $V_{D}=$ (dashed line) 0 , (thin line) $0.1 \mathrm{v}$, and (thick line) $-0.1 v$ 


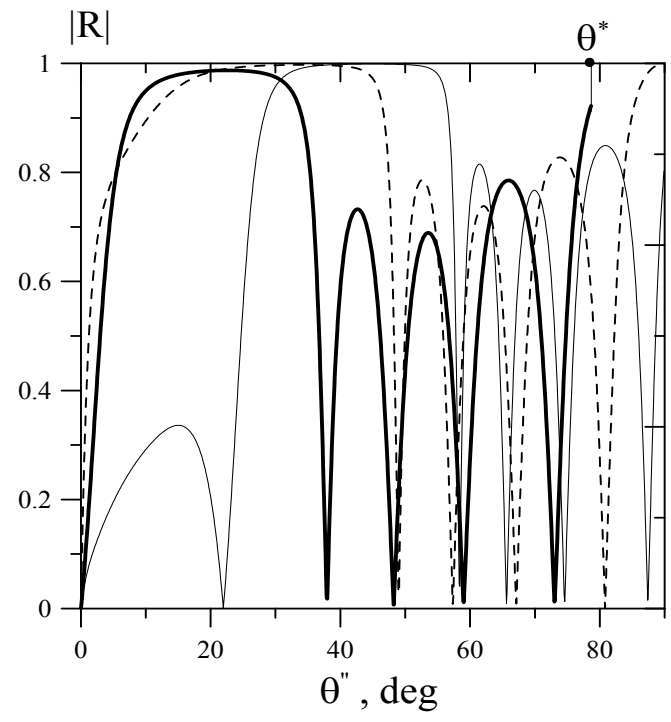

Fig. 9. Dependences of the magnitude of the reflectance $|R|$ on the angle of incidence $\theta^{\prime \prime}$ for the structure " ++ " consisting of nine domains $(\mathrm{N}=4)$ in the case of $k^{\prime \prime}=31941 \mathrm{sm}^{-1}$ at the velocities $V_{D}=$ (dashed line) 0 , (thin line) $0.1 v$, and (thick line) $-0.1 v$

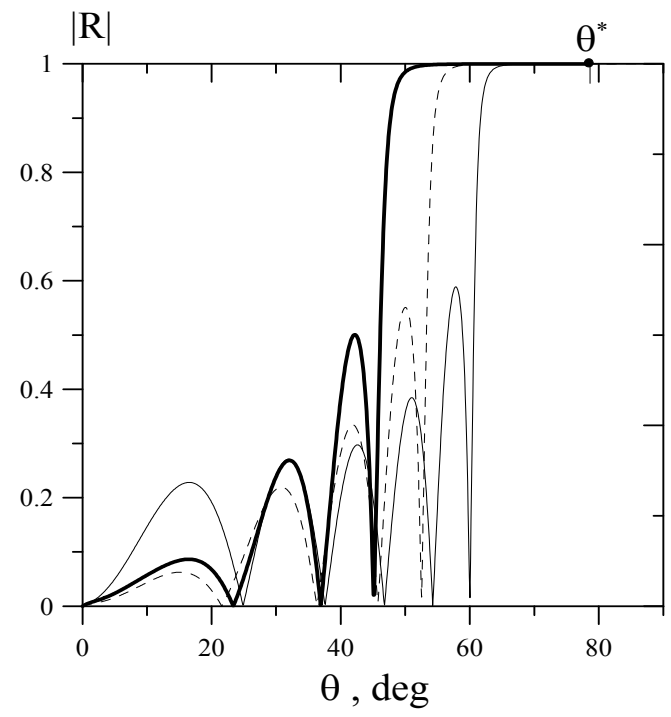

Fig. 10. Dependences of the magnitude of the reflectance $|R|$ on the angle of incidence $\theta^{\prime \prime}$ for the structure "++" consisting of five domains $(\mathrm{N}=2)$ in the case of $k^{\prime \prime}=55000 \mathrm{~cm}-1$ at the velocities $V_{D}=$ (dashed line) 0 , (thin line) $0.1 v$, and (thick line) $-0.1 v$ 
with the wavelength considerably larger than $2 \mathrm{Nd}+d$ is "insensitive" to the structure ",++ , and the electroacoustic wave propagates in the ferroelectric as in a single-domain sample in which the reflection is absent; i.e. $|\mathrm{R}| \rightarrow 0$ at $k \rightarrow 0$ (Fig. 7). For the electroacoustic wave with the wavelength $\lambda \rightarrow \infty$, the structure "+-" is represented as a single domain wall, which is confirmed by the results of numerical calculations (Fig. 8).

The second difference lies in the fact that, at the center of the allowed band, the reflectance spectrum for the structure " ++ " is characterized by $|R|=0$, whereas the reflectance spectrum for the structure "+-" always contains the secondary maximum. Finally, the third evident difference manifests itself in the number of peaks (the main peak plus secondary peaks) in one band gap: their number is always odd for the structure "++" and always even for the structure "+-."

The dependences of the magnitude of the reflectance $|R|$ of electroacoustic waves on the angel of incidence $\theta$ " of the electroacoustic wave on the lattice for the structure "++" are plotted in Figs. 9 and 10. It can be seen from Fig. 9 that, for the wave number corresponding to the center of the first band gap, it is possible to choose the condition providing an almost total reflection $(|R| \approx 1)$ in the range of small angles of incidence. The domain-wall motion leads to the fact that the reflectance peaks shift toward smaller (larger) angles of incidence for the lattice moving away (approaching) as compared to the reflectance peaks for the static lattice. In this case, the number of Bragg maxima for the approaching lattice increases by unity. It can also be seen that, for the lattice moving away, the dependence represented by the thick curve, according to condition (32) is limited by the angles of incidence corresponding to $\theta^{\prime \prime}<\theta^{*}$. When the condition $k_{x} d>>1$ is satisfied, the magnitude of the reflectance reaches unity even in the range of grazing angles of incidence, which can be clearly seen from Fig. 10. It should be noted that the strict fulfillment of the inequality $\exp \left(-k_{x} d\right)<<1$ represents the condition for the existence of the Marfeld-Tournois mode at the outer surface of the domain-wall lattice [Maerfield \& Tournois, 1971].

\section{Electroacoustic waves confined by a moving domain wall superlattice of a ferroelectric crystal}

\subsection{The statement of the problem}

For completeness of the description of influence of motion of domain boundaries on a spectrum of electroacoustic waves, there is a necessity of consideration of propagation of electroacoustic interfacial waves boundary guided by a DW in a ferroelectric with a lattice of moving domain boundaries. In this section the dispersion properties of electroacoustic wave modes confined by a superlattice of $180^{\circ}$ domain walls uniformly moving in a tetragonal ferroelectric crystal are considered. The crystal symmetry, wave polarization type, and the propagation geometry are the same as in [Golenishchev-Kutuzov et al, 2003; Li et al, 1991; Lyubimov \& Sannikov, 1979], the only additional assumption being that the consideration is restricted to modes localized on the DWs of the superlattice.

Geometry of the problem is presented in Fig. 11, where the dynamic superlattice of equidistant $180^{\circ}$ DWs with the (010) orientation is shown in the accompanying frame of reference $\tilde{x} 0 \tilde{y} \tilde{z}$. In virtue of the inequality $V_{D}<<$, where $\mathrm{c}$ is the speed of light and $V_{D}$ is the velocity of DW motion, this frame is related to the laboratory one $x 0 y z$ via the Galilean transformation (11). As the main structure-forming element of the supperlattice, chose a pair 
of adjacent stripe domains of a ferroelectric with the interfaces $\tilde{y}=0, d$, and $2 d$ shown in Fig. 11 with dark. The rest domains are formed by translation of this pair to whole number $n$ of lattice periods $2 d$ in the positive $(n>0)$ or negative $(n<0)$ direction of the $\tilde{y}$. axis. Ascribe the number $j=1$ to the lower domain of the pair of a lattice unit cell at $0<\tilde{y}<d$ and the number $j=2$ to the upper domain at $d<\tilde{y}<2 d$. Thus, each domain of the lattice will be defined by the translation number $n=0, \pm 1, \pm 2, \pm 3, \ldots$ and the pair number $j=1$ or $j=2$; the value $n=0$ corresponds to the unit (initial) cell.

In view of the aforesaid, under the conditions of specified DW motion at the velocity $V_{D}$ $\|\mathrm{y}\|[010]$ the current coordinates in the laboratory frame are $y_{n}=V_{D} t+n d$. Considering that antiparallel polarizations in domains of the lattice unit cell are related to the sign alteration of piezoelectric modulus $e_{15}$, which is the only active one under the given conditions (9). Extend the validity of this condition over the entire lattice, assuming that in Eq. (9) we have

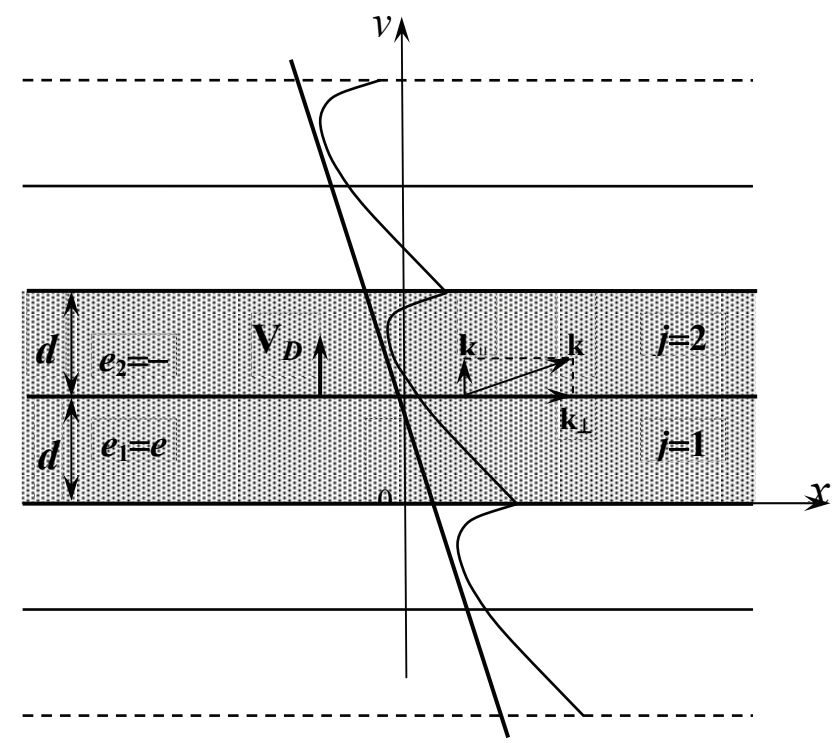

Fig. 11. Schematic diagram showing geometry of the problem and the distribution of shear displacements along the tilted front of a partial EIW with $\kappa=\pi / d$

$$
j=\left\{\begin{array}{c}
1, \text { for } n d+V_{D} t<y<(n+1) d+V_{D} t \\
2, \text { for }(n+1) d+V_{D} t<y<(n+2) d+V_{D} t
\end{array} .\right.
$$

The lattice cell fields are described by the solutions of the equations (13)-(14). Then, assuming that the waves propagate in the plane $\tilde{x} 0 \tilde{y}$ in the positive direction of the $\tilde{x}$ axis, with regard of proportional displacements $u_{j}$ and potentials $\Phi_{j}$ with the exponential coefficient $\exp \left[i\left(k_{\mid} \tilde{x}-\Omega \tilde{t}\right)\right]$, we conclude that the EIWs are noncollinear: $\mathbf{k}=\left(k_{\mid} \mid, k_{\perp}, 0\right), k_{\perp} \neq 0$ (Fig. 11). Deviation of the EIW front from the orthogonal position is determined by the transverse component of wave vector $k_{\perp}$ [Gulyaev et al, 2000]: 


$$
k_{\perp}=\frac{\Omega}{c_{t}} \frac{\beta}{1-\beta^{2}},
$$

where $\beta=\mathrm{V}_{D} / c_{t}$.

Assume also that the EIW length is much less than the characteristic size of a crystal. Under such conditions, the interfacial effects at the external interfaces of a ferroelectric, as well as the shape of the latter, will not have a considerable effect on the behavior of the electroacoustic waves and can be disregarded.

\subsection{Derivation of the EIW dispersion equation for the dynamic lattice of a ferroelectric crystal}

Solutions of Eqs. (13)-(14) within the pair of domains numbered with the indices $j=1$ and 2 of the unit cell can be presented as

$$
\begin{gathered}
u_{1}(\tilde{y})=\left[A_{1} \exp (-s \tilde{y})+A_{2} \exp (s \tilde{y})\right] \exp \left(i k_{\perp} \tilde{y}\right) \exp \left[i\left(k_{\mid} \tilde{x}-\Omega \tilde{t}\right)\right], \quad(0<\tilde{y}<d), \\
\Phi_{1}=\left[C_{1} \exp \left(k_{\mid} \tilde{y}\right)+C_{2} \exp \left(-k_{\mid} \tilde{y}\right)\right] \exp \left[i\left(k_{\mid} \tilde{x}-\Omega \tilde{t}\right)\right], \\
u_{2}(\tilde{y})=\left[B_{1} \exp (-s \tilde{y})+B_{2} \exp (s \tilde{y})\right] \exp \left(i k_{\perp} \tilde{y}\right) \exp \left[i\left(k_{\mid} \tilde{x}-\Omega \tilde{t}\right)\right], \quad(d<\tilde{y}<2 d), \\
\Phi_{2}=\left[D_{1} \exp \left(k_{||} \tilde{y}\right)+D_{2} \exp \left(-k_{\mid} \tilde{y}\right)\right] \exp \left[i\left(k_{\mid} \tilde{x}-\Omega \tilde{t}\right)\right],
\end{gathered}
$$

where $\Omega=\omega\left(1-\beta^{2}\right)$ is the EIW frequency in the accompanying frame of reference and $s$ is the coefficient of interfacial localization

$$
s=\frac{1}{1-\beta^{2}} \sqrt{k_{||}^{2}\left(1-\beta^{2}\right)-\frac{\Omega^{2}}{c_{t}^{2}}},
$$

related to frequency $\omega$ in the laboratory frame by the formula $\omega=c_{t} \sqrt{k_{||}^{2}\left(1-\beta^{2}\right)^{-1}-s^{2}}$. To sew the domain fields at the internal interface $\tilde{y}=d$, we will use the standard conditions (20) of continuity of shear displacements, potentials, shear components of the stress tensor $T_{y z}(j)$, and $y$-components of the electric inductions .

For fields with structure of type (44), (46) procedure of construction of a matrix of transition and the dispersive equation will be same, as well as for waves of bulk propagation (see section 3 and 4), but there are the replacements in (13), (18) - (21) $\tilde{y}, \tilde{x}$ - directional derivative according Eqs (44), (46). We do not present the intermediate calculations here and at once write down the dispersion relation of EIWs in the following form:

$$
\lambda^{4}+\lambda^{3} Q\left(k_{||}, s\right)+\lambda^{2} P\left(k_{||}, s\right)+\lambda R\left(k_{\mid}, s\right)+\frac{1}{\delta^{2}}=0,
$$

where the eigenvalues $\lambda=\exp (2 i \kappa d)$ of matrix of transition and $\kappa$ is the Bloch wavenumber characterizing the degree of phase synchronism of the electroacoustic oscillations on DWs over the lattice period. At the specified geometric and material parameters, quantities $Q, P$, and $R$ serving as coefficients in Eq. (47) represent the following functions of spectral variables $k_{\|}$and $s$ : 


$$
\begin{gathered}
Q\left(k_{||}, s\right)=-2 \operatorname{ch}(2 s d) \delta^{-1}-2 \operatorname{ch}\left(2 k_{||} d\right)+\frac{8 K^{2} k_{||} \operatorname{sh}(s d) \operatorname{sh}\left(k_{||} d\right)}{s \delta^{1 / 2}}, \\
P\left(k_{\mid}, s\right)=1+\frac{1}{\delta^{2}}+\frac{16 K^{2} k_{||}}{s}\left[\operatorname{sh}\left(k_{||} d\right) \operatorname{sh}(s d)\left(\frac{K^{2} k_{||}}{\delta s} \operatorname{sh}\left(k_{||} d\right) \operatorname{sh}(s d)+\delta^{1 / 2}(1+\delta)\right)\right]+ \\
+\frac{4 \operatorname{ch}\left(2 k_{||} d\right) \operatorname{ch}(2 s d)}{\delta}-\frac{8 K^{2} k_{||} \operatorname{sh}(2 s d) \operatorname{sh}\left(2 k_{||} d\right)}{\delta s}, \\
R\left(k_{||,}, s\right)=-2 \frac{\operatorname{ch}(2 s d)}{\delta}-\frac{2 \operatorname{ch}\left(2 k_{||} d\right)}{\delta^{2}}+\frac{8 K^{2} k_{||} \operatorname{sh}(s d) \operatorname{sh}\left(k_{\mid} d\right)}{\delta^{3 / 2}} \\
\delta=\exp \left(-2 i k_{\perp} d\right) .
\end{gathered}
$$

The roots of Eq. (47), along with expressions (48), relate spectral EIW parameters $k_{||}$and s to the Bloch wavenumber. Holding to the interpretation of the dispersion law as the functional coupling $s=s\left(k_{||}\right)$accepted in the theory of electroacoustic waves [Maerfield \& Tournois, 1971 and Gulyaev, 1969], it would be reasonable to consider Bloch wavenumber $\kappa$ as an additional parameter. The occurrence of this parameter owes to the conditions of translational symmetry of the solution describing EIWs over the entire set of allowed values $-\pi / d<\kappa<\pi / d$. Thus, at the designated $\kappa$ value, Eq. (48) along with expressions (49) establish the dispersion properties of a partial EIW.

Features of a spectrum of the partial EIWs confined by a moving lattice are determined by the velocity of DW motion. In Equation (47), the parametric dependence on $V_{D}$ is implicit and implemented through wave vector component $k_{\perp}$ from (43). In view of inconvenience of the obtained expressions and, above all, transcendence of Eq. (47), the dependences of the EIW dispersion spectra on the velocity of DW motion can be demonstrated only numerically. However, multiplying Eq. (47) by $\delta$ and dividing it by $\lambda^{2}$ in accordance to Eq. (48), we obtain the simpler form:

$$
\begin{aligned}
& a^{2}+a\left[4 \cos \left(k_{\perp} d\right)+4 \cos \left(2 \kappa d-k_{\perp} d\right)-8 \operatorname{ch}(s d) \operatorname{ch}\left(k_{||} d\right)\right]+\cos \left(4 \kappa d-2 k_{\perp} d\right)-4 \cos (2 \kappa d) \operatorname{ch}(2 s d)- \\
& -4 \cos \left(2 \kappa d-2 k_{\perp} d\right) \operatorname{ch}\left(2 k_{||} d\right)+2 \cos \left(2 k_{\perp} d\right)+4 \operatorname{ch}(2 s d) \operatorname{ch}\left(2 k_{||} d\right)=0,
\end{aligned}
$$

where $a=4 k_{||} K^{2} \operatorname{sh}(\mathrm{s} d) \operatorname{sh}\left(k_{||} d\right) / s$.. Then, solving Eq. (49) with respect to $a$, one can obtain a dispersion relation in the form more convenient for an analysis and numerical calculations. In particular, if the Bloch wavenumber $\kappa=\pi /(2 d)$ is taken (in the periodic structure only the $\kappa$ values lying within the first allowed band $-\pi / d<\kappa<\pi / d$ correspond to the physically nonequivalent states), from Eq. (49) we obtain

$$
s=K^{2} k_{||}\left\{\operatorname{th}\left(k_{||} d\right) \operatorname{th}(s d) \frac{\left[1 \pm \sin ^{2}\left(k_{\perp} d\right) / \operatorname{ch}(s d)\right]}{\left[1-\sin ^{2}\left(k_{\perp} d\right)\right] / \operatorname{ch}^{2}(s d)}\right\} .
$$

Expression (50) shows, in particular, that while for a static lattice $\left(k_{\perp}=0\right)$ the only mode exists, in the case of a moving lattice $\left(k_{\perp} \neq 0\right)$ the spectrum splits into two different modes corresponding to the alternating " + " and "- signs. This splitting can be considered as the 
removal of a degeneracy of the interaction of acoustoelecric oscillations of DWs of a unit cell due to their transverse motion taking place for $\kappa=\pi /(2 d)$.

To turn to the static lattice at $\kappa \neq \pi /(2 d)$, one should take $k_{\perp}=0$ in Eq. (49). After solving the quadratic equations with respect to $a$ and making some transformations, we obtain the explicit representation of the EIW dispersion equation:

$$
s=-k_{\mid} K^{2}\left\{\frac{\operatorname{sh}\left(k_{||} d\right) \operatorname{sh}(s d)}{\left[\cos (\kappa d) \mp \operatorname{ch}\left(k_{||} d\right)\right][\cos (\kappa d) \pm \operatorname{ch}(s d)]}\right\} .
$$

According to Eq. (51), the partial EIW spectrum in a ferroelectric with the static lattice initially includes two modes for a fixed value of the Bloch wavenumber different from $\pi /(2 d)$. This result could be expected from the results reported in [Lyubimov \& Sannikov, 1979] for a stripe domain. Note, however, that at finite values of the lattice period no strict correspondence between the spectra of modes (49) and (51) in the limit $V_{D} \rightarrow 0$ and the spectra from [Lyubimov \& Sannikov, 1979] is observed due to the conditions of translational symmetry of solution (11). On the other hand, the transition to the results of [Lyubimov \& Sannikov, 1979] under the condition $d \rightarrow \infty$ is excluded from Eqs. (49) and (51) at all, as it requires additional consideration of another boundary problem for the structure of alternating domains with different thicknesses. The only available analytical confirmation of correspondence remains the fact that in the limit $d \rightarrow \infty$, when the transition to an individual DW occurs, from Eqs. (49) and (51) we immediately have the expected result $s=k_{||} K^{2}$ [Maerfield \& Tournois, 1971].

\subsection{Dispersion spectra of modes of noncollinear electroacoustic interfacial waves of a dynamic superlattice of a ferroelectric crystal}

It is reasonable to start the study of spectra of the partial EIWs of a dynamic superlattice with the case $\kappa=\pi / d$, when the electroacoustic oscillations of DWs distant from one another by a lattice period ( $\lambda_{B}=2 d$ is length of Bloch wave) are inphase. One can see from Eq. (49) that the results for this case are identical to those for $\kappa=0$ and $\kappa=-\pi / d$. Another feature is that the transition to the case $\kappa=-\pi / d$ can be implemented by inversion of the velocity of DW motion: $V_{D} \rightarrow-V_{D}$. Physically, it means that the dispersion spectra of EIW modes under the condition of synchronism of the electroacoustic oscillations of DWs are independent of the chosen direction of DW motion; i. e., the lattice reveals reciprocity of the transverse distribution of fields and EIW propagation.

The values of Bloch wavenumber $\kappa= \pm \pi / d$ determine the boundaries of the first allowed band. A schematic of the dispersion EIW spectra for the case $\kappa= \pm \pi / d$ is presented in Fig. 12 . The dispersion branches showed by dashed lines correspond to the static lattice; the presence of DW motion is reflected by bold solid lines. Thin straight lines represent the linear EIW spectra on a single DW, static $(\beta=0)$ [Maerfield \& Tournois, 1971] or moving $(\beta \neq 0)$ [Gulyaev et al, 2000]. A dashed straight line shows the linear spectrum of volume SH waves propagating in a single-domain crystal.

The overall picture of the spectrum of modes of the partial EIWs at $\kappa= \pm \pi / d$ outwardly resembles the picture of the spectrum of modes of a stripe domain: there are only two modes and the high-frequency one has a lower cutoff frequency (black point in the figure); the low-frequency mode is present over the entire frequency range. The change in the highfrequency asymptotes of the spectra due to DW motion is the same as that for a single DW 
[Gulyaev et al, 2000] or a stripe domain [Bugaev et al, 2005]. However, there are principle distinctions between the spectra of stripe domain modes and the spectra in Fig. 12.

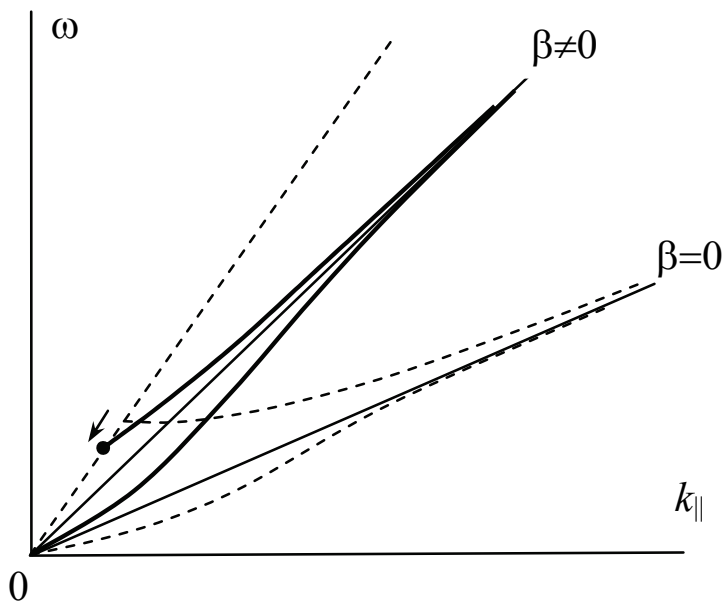

Fig. 12. General view of the mode spectrum of the partial EIW for the lattice with the Bloch wavenumbers $\kappa= \pm \pi / d$

First, as the velocity of DW motion increases, the cutoff point of the high-frequency mode in Fig. 12 shifts, as the arrow indicates, along the spectrum of volume waves towards lower frequencies, i. e., in the opposite direction as compared to the case of a moving stripe domain. A consequence of this behavior is the limiting situation at $\beta \rightarrow 1$, when the high- and low-frequency branches merge with the linear spectrum of volume waves (the Marfeld-Turnois asymptote at $\beta \rightarrow 1$ approaches the volume wave spectrum) and the region of EIW existence degenerates into the spectral line. This degeneracy of the EIW into an ordinary shear wave propagating in the direction of DW motion corresponds to "tripping" the shear waves and the moving DWs due to the absence of piezoelectric polarization charges [Gulyaev et al, 2000]. This result is quite expectable, since the number of DWs moving at the sound velocity is of no importance, as in the case of the superlattice.

Second, the low-frequency branch exhibits a qualitatively different character of dispersion. Indeed, at low frequencies the dispersion branch for a stripe domain occurs below the spectrum of volume waves but above the Marfeld--Turnois mode asymptote. In other words, the phase velocity of the wave lies between the values of the EIW velocity on a single $\mathrm{DW}$ and the velocity of a volume $\mathrm{SH}$ wave. Meanwhile, in the lattice spectrum the dispersion branch of the low-frequency mode lies completely below the Marfeld--Turnois asymptote. This type of the EIWs for a static lattice was mentioned previously in [Li, et al, 1991], where the resemblance of their dispersion to the dispersion of flexural modes in thin planes was pointed out.

Third, the mode spectra of a stripe domain correspond to different types of the transverse distribution of shear displacements, including symmetric for the low-frequency branch (symmetric mode) and antisymmetric for the high-frequency branch (antisymmetric mode). In the case of a lattice, the calculations using formulas (51) with the dispersion EIW indices obtained from Eq. (47) show that the distributions of the shear displacements across the unit 
cell are qualitatively similar for both modes and correspond mainly to the symmetric type. Obviously, the DW motion causes the symmetry break. Figure 11 demonstrates such a quasi-symmetric profile shown, for better illustration, in a strongly emphasized form.

When there are only two modes at $\kappa= \pm \pi / d$, the degenerated case of EIW propagation takes place, i. e., there is no splitting of modes upon induced DW motion. Possibility of this splitting in other cases was analytically demonstrated in the previous section by the example of the lattice with $\kappa=\pi /(2 d)$. The shape of the partial EIW mode spectrum for the lattice with this Bloch wavenumber calculated from Eq. (50) is presented in Fig. 13.

As was mentioned above, in the static case Eq. (50) has the only root. The corresponding mode is shown in Fig. 13 by a dashed curve and is purely antisymmetric judging by the character of the transverse distribution of the shear displacements. This naturally follows from the fact that at $\kappa=\pi /(2 d)$ the electroacoustic oscillations on DWs distant by a lattice period are antiphase. The absence of a symmetric mode is explained by piezoelectric inconsistency of the symmetrically distributed shear displacements and the resulting stresses in an internal DW of the unit cell due to the alteration of a sign of the piezoelectric modulus [Li, et al, 1991].

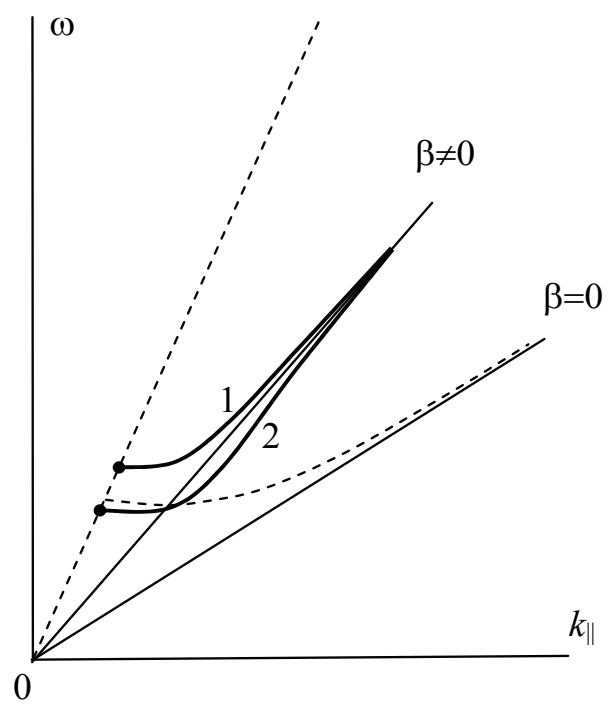

Fig. 13. Spectrum of the partial EIW modes for the Bloch wavenumber $\chi=\Pi(2 d)$ : 1 and 2 are the branches split by DW motion

Motion of DWs splits the dashed branch of the antisymmetric mode into two branches 1 and 2 shown in Fig. 13 by bold solid lines. In the high-frequency limit, the spectra of the split modes are asymptotically drawn to the changed (turned towards the dashed line of the volume wave spectrum) Marfeld--Turnois asymptote. With the change in the direction of DW motion (alteration of signs in Eq. (50) from \pm to $\mp$ ), branches 1 and 2 in Fig. 13 change places. Clearly, this does not break the overall picture of the spectrum. In this sense, the conclusion on reciprocity of the transverse field distribution and EIW propagation revealed by the lattice of moving DWs retains its validity in the considered variant. 
One can see from Eq. (50) that the effect of the inversion of the velocity of DW motion on the spectrum of modes of the partial EIW is equivalent to that of the transformation $\kappa \rightarrow-\kappa$. Thus, the picture of the spectra in Fig. 13 includes the case $\kappa=-\pi /(2 d)$. This conclusion is general and can be extended to any case $\kappa \neq \pi /(2 d)$. The most typical situation of the transformation of the partial EIW mode spectrum by the lattice of moving DWs is that for $\kappa \neq \pi /(2 d)$ in the static case there are already two modes (dashed curves in Fig. 14) instead of the only mode split into pairs by motion, as in Fig. 13. The transformations $V_{D} \rightarrow-V_{D}$ or $\kappa \rightarrow$ $-\kappa$ yield intrapair rearrangement of the split modes $1 \leftrightarrow 2$ and $3 \leftrightarrow 4$ (solid curves), which validates general independence of spectra representation on the direction of DW motion.

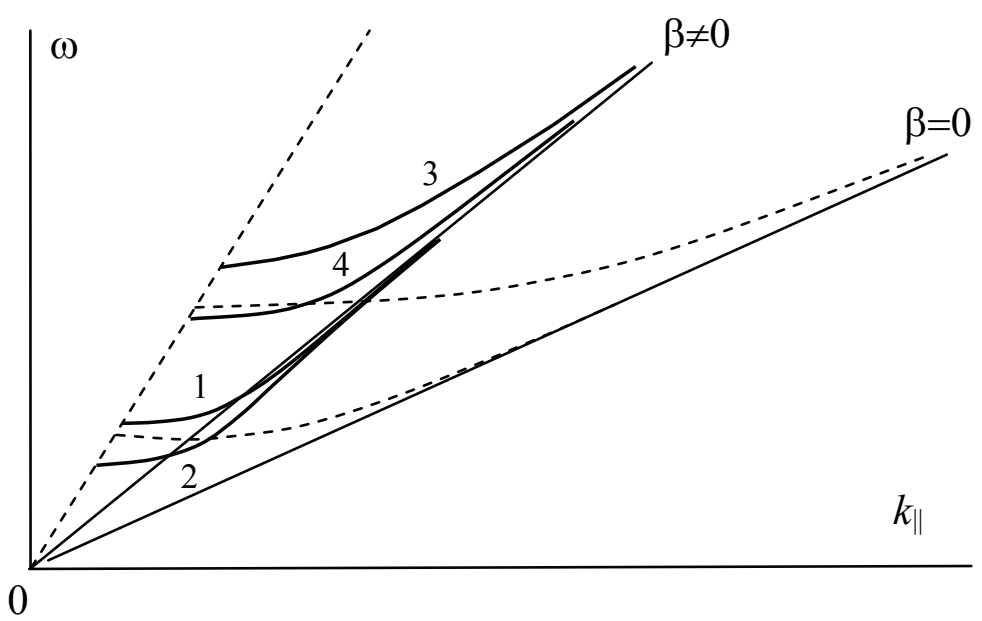

Fig. 14. Spectrum of the partial EIW modes for the arbitrary Bloch wavenumber $\kappa \neq \pi /(2 d)$ and $\kappa \neq \pi / d: 1,2$ and 3,4 are the pair branches split by DW motion

Concerning the structure of the transverse distribution of the shear displacements for the split branches, one should pay attention on its noticeable closeness to the transverse distribution for a generative mode of a partial EIW of the static lattice. In the general case, the generative mode reveals mixed signs of symmetry-antisymmetry occurring depending on closeness of value $2 \kappa d$ to values $2 \pi$ or $\pi$. If the situation corresponds to that illustrated in Fig. 13, the transverse distributions of the displacements for both split modes slightly differ from a strictly symmetric form at high velocities of DW motion $(\beta>0.7)$ and from one another.

Figure 15 depicts the dependence of the frequency of an electroacoustic wave on the Bloch wavenumber for some fixed wavenumber. The dispersion curves shown by dashed lines correspond to the static lattice; in the presence of DW motion, they are shown by solid lines. The low-frequency group of curves 1 corresponds just to the EIWs. The high-frequency group of curves 2 separated from group 1 by the band gap corresponds to the first allowed band for the electroacoustic waves of volume propagation [Vilkov, 2008] (presented for comparison). There is the only allowed band for the EIWs; it lies below all the allowed bands of the volume propagation spectra. In both cases, DW motion splits the spectrum of any electroacoustic wave of the static lattice into pairs (spectral doublet). In the general case 
$\kappa \neq \pi /(2 d)$ and $\kappa \neq \pi / d$ for the EIWs at the chosen Bloch number and wave vector we have four branches, as not one but two modes corresponding to different symmetries of the distribution of the shear displacements across the cell undergo pair splitting ${ }^{2}$. One can see from Fig. 15 that although the values of the Bloch number $\kappa= \pm \pi / d$ determine the boundaries of the first allowed band, they do not determine the frequency region of location of the dispersion branches of all the rest partial EIWs. There exist intermediate Bloch wavenumbers for which the frequency at a fixed wavenumber lies above or below as compared to the case of the Bloch wavenumber $\kappa= \pm \pi / d$. This feature is more pronounced for the dynamic lattice.

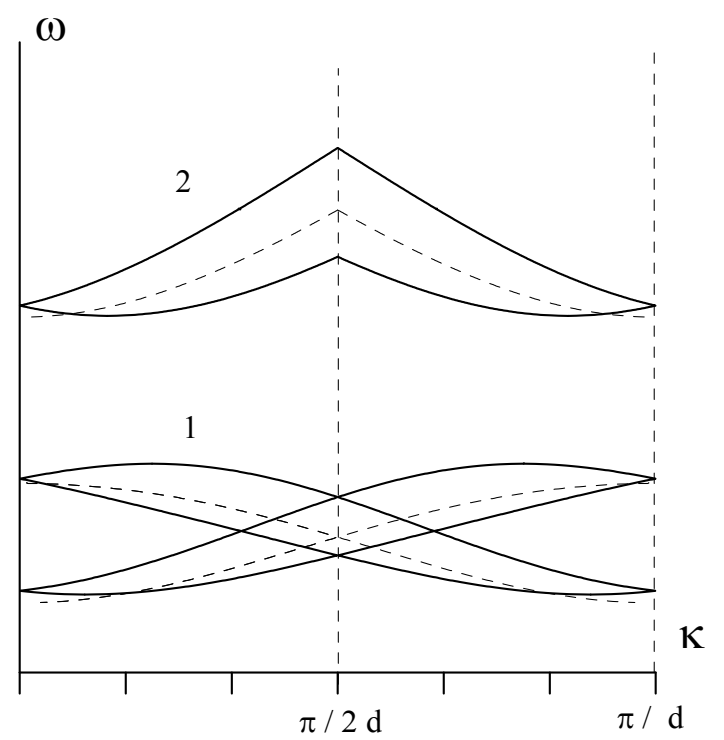

Fig. 15. Dependence of the frequency of an electroacoustic wave on the Bloch wavenumber at a fixed $k$ value

\section{Conclusions}

The effect of the uniform motion of ferroelectric DWs that form a dynamic superlattice on the spectral properties of EWs has been analyzed for the first time. The results obtained indicate that even slow DW motions cause significant (detectable) variations in the wave spectra. Moreover, the spectrum of reflection of electroacoustic waves from a periodic domain structure formed by a finite number of uniformly moving 180-domain walls in the tetragonal ferroelectric was analyzed. It was established that, owing to the interference of reflected waves, the magnitude of the reflectance for the wave number corresponding to the band gap of the Bloch spectrum tends to unity for both static and moving domain structures. The inclusion of the domain-wall motion leads to shift of the Bragg reflectance

\footnotetext{
${ }^{2}$ For the regions not close to $\kappa=\pi /(2 d)$, and $\kappa=\pi / d$, two solid curves lying above in frequency correspond to the quasi-symmetric distribution.
} 
peaks toward the long-wavelength (short-wavelength) range in the case of the counter (oneway) motion of domain walls. The higher the velocity of domain-wall motion, the larger the shift of the peaks. The intensity of these peaks decreases (increases) as compared to that in the case of static domain walls. It was demonstrated that, apart from the appropriate choice of the lattice parameters and the wave characteristics of electroacoustic waves, the range of the total reflection at small angles of incidence and those close to grazing angles of incidence can be controlled by specifying the velocity of domain walls.

In the chapter the dispersion properties of the noncollinear EIWs of the dynamic superlattice of the equidistant uniformly moving $180^{\circ}$ DWs of ferroelectric crystals have been also described. It has been shown that due to DW motion the partial Bloch spectra of modes of the interfacial electroacoustic waves which are not related to the boundaries of the first allowed band undergo pair splitting into the high- and low-frequency branches changing places during the inversion of the DW velocity. The partial modes setting the limits of the allowed band for the EIWs are not subjected to the mentioned splitting, invariant to the inversion of the velocity of DW motion, and transformed by DW motion in the highfrequency asymptote similar to the waves on individual domain walls.

It was revealed that the velocity of domain-wall motion serves as a new parameter that is convenient for controlling the reflection and transmission of waves in combination with their frequency shifts. This circumstance leads to additional experimental possibilities of the acoustic spectroscopy of polydomain ferroelectrics by means of the application of the acoustic nonreciprocity of the crystals that is induced by DW motion. The induced acoustic nonreciprocity and the Doppler frequency conversion can be used for the development of sensors and acoustoelectronic devices for data conversion with the frequency output.

\section{References}

Auld B.A. (1973). Acoustic Field and Waves in Solids Vol.2, Wiley-Interscience, ISBN 0471037028, New York.

Balakirev, M. K. \& Gilinskii, I. A. (1982). Waves in Piezoelectric Crystals, Nauka, ISBN 5-BOB-БА-82, Novosibirsk.

Bass, F.G., Bulgakov, A. A., \& Tetervov A. P. (1989). High-Frequency Properties of Semiconductors with Superlattices, Nauka, ISBN 5-02-014021-X, Moscow.

Batanova, N.L. \& Golenishchev-Kutuzov A.V (1997). Acoustic Waves in Ferro- and Piezoelectrics with a Periodic Electrical Relief. J. Acoust. Phys. Vol.43, No 4, pp.468471, ISSN 1063-7710.

Bugaev, A. S., Maryshev S. N., \& Shevyakhov N. S. (2005) Electroacoustic Waves at the Interfaces between Two Strip Domains Moving in a Ferroelectric. // Journ. Communications Technol. and Electronics. Vol. 50. No 9. pp. 1031-1037, ISSN 1064-2269.

El'meshkin, O. Yu., \& Shevyakhov, N.S. (2006) Mode spectrum of noncollinear electroacoustic boundary-guided waves in a ferroelectric with a moving strip domain J. Acoust. Phys. Vol.43, No 1, pp. 56-61, ISSN 1063-7710

Esayan, S. Kh., Lemanov, V. V., \& Smolenskiŭ G. A. (1974) Reflection and refraction of elastic waves on the domain boundaries in ferroelectric crystals $\mathrm{Gd}_{2}\left(\mathrm{MoO}_{4}\right)_{3}$. Sov. Phys. Dokl., Vol 19, No 7, pp. 393-401.

Fabelinskii, I. L. (1968). Molecular Scattering of Light. PLenum Press, New York. 
Golenishchev-Kutuzov, A. V., V. A. Golenishchev-Kutuzov, V. A., and R. I. Kallimullin, (2003) Induced Domain Structures in Electrically and Magnetically Ordered Materials, Fizmatlit, ISBN: 978-5-9221-0449-4 Moscow.

Gulyaev, Yu. V. (1969) Electroacoustic surface waves in solids. JETP Lett., Vol. 9, No 1 pp. 37-38, ISSN 0021-3640.

Gulyaev, Yu. V., El'meshkin, O. Yu., \& Shevyakhov N. S. (2000) Electroacoustic surface waves on moving boundaries // Journ. Communications Technol. and Electronics. Vol. 45. No 3. pp. 351-356, ISSN 1064-2269

Korn, G.A. \& Korn, T.M. (1968), Mathematical Handbook for Scientist and Engineers, Second Edition, McGraw- Hill Book Company, ISBN 10: 0-07-035370-0, ISBN 13: 9780070353701, Inc., New York.

Li, Xingjiao. (1987.) Analysis of the field of the ferroelectric domain layer mode. Journ. Appl. Phys., Vol. 61. No 6. pp. 2327-2334, ISNN 0021-8979.

Li, Xingjiao; Li, Yibing; Lei, Yiwu \& Cross, L. E. (1991). The ferroelectric domain layer interface wave in multiple domain layered structure. Journ. Appl. Phys., Vol. 70, No 6, pp.3209-3214, ISSN 0021-8979.

Lyubimov, V. N. \& Sannikov D. G. (1979) Surface acoustic waves on 180-degree domain boundaries in ferroelectrics of type barium titanate crystal. Kristallografiya, Vol 24 No 1, pp.5-10, ISSN 0023-4761. .

Maerfield C. \& Tournois P. (1971) Pure Shear Elastic Surface Wave Guided by the Interface of Two Semi-Infinite Media. Appl. Phys. Lett., Vol. 19, No 4. pp. 117-118, ISSN 00036951

Nougaoui A., Rouhani, B.Djafari. (1987) Dynamics of infinite and semi-infinite piezoelectric superlatticies: shear horizontal waves and effective medium approximation. Surf. Sci., Vol. 185. No 1-2. pp. 154-174.

Shevyakhov, N. S. (1990) Reflection of a transverse wave by a moving domain wall in a tetragonal ferroelectric. Akust. Zh. Vol. 36, No 1, pp.160-165, ISSN 0320-7919.

Shenderov E.L. (1997) Reflection of a plane sound wave from a semi-infinite periodic transversely isotropic set of layers. J. Acoust. Soc. Am., Vol. 101, No 3, pp.12391249, ISSN 0001-4966.

Shuvalov A.L. \& A.S. Gorkunova A.S. (1999) Transverse acoustic waves in piezoelectric and ferroelectric antiphase superlattices. Phys. Rev. B, Vol. 59, No 14, pp.9070-9077.

Sosnin, A. S. \& B. A. Strukov. (1970) Introduction to Ferroelectricity, Vysshaya Shkola, Moscow.

Vainshtein L. A. (1988). Electromagnetic Waves, Radio i Svyaz, ISBN 5256000640, Moscow.

Vilkov, E.A., (2007) Spectrum of magnetostatic waves in a ferromagnet with a moving superlattice of domain walls. Physics of the Solid State, Vol. 48, No 9, pp. 1754-1759, ISSN 1063-7834.

Vilkov, E.A. (2008) Spectral properties of electroacoustic waves in a ferroelectric with a moving periodic domain structure. Physics of the Solid State, Vol. 50, No 8, pp. 15191526, ISSN 1063-7834.

Vilkov, E.A. (2009) Reflection of electroacoustic waves from a system of moving domain walls in a ferroelectric. Physics of the Solid State, Vol. 51, No 2, pp. 343-350, ISSN 1063-7834. 
Vilkov, E.A., Maryshev S.N. \& Shevyakhov (2009) N.S. Electroacoustic waves of a moving domain wall superlattice in a ferroelectric crystal. Technical Physics Letters, Vol. 35, No 4, pp.326-330, ISSN 1063-7850. 


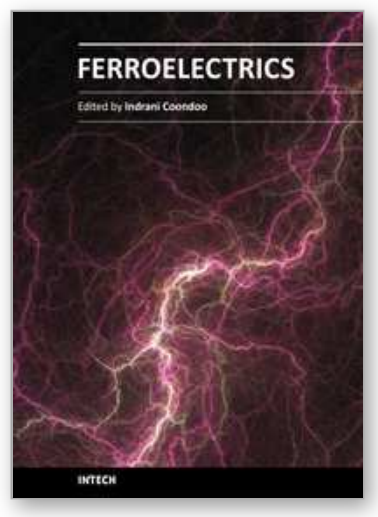

\author{
Ferroelectrics \\ Edited by Dr Indrani Coondoo
}

ISBN 978-953-307-439-9

Hard cover, 450 pages

Publisher InTech

Published online 14, December, 2010

Published in print edition December, 2010

Ferroelectric materials exhibit a wide spectrum of functional properties, including switchable polarization, piezoelectricity, high non-linear optical activity, pyroelectricity, and non-linear dielectric behaviour. These properties are crucial for application in electronic devices such as sensors, microactuators, infrared detectors, microwave phase filters and, non-volatile memories. This unique combination of properties of ferroelectric materials has attracted researchers and engineers for a long time. This book reviews a wide range of diverse topics related to the phenomenon of ferroelectricity (in the bulk as well as thin film form) and provides a forum for scientists, engineers, and students working in this field. The present book containing 24 chapters is a result of contributions of experts from international scientific community working in different aspects of ferroelectricity related to experimental and theoretical work aimed at the understanding of ferroelectricity and their utilization in devices. It provides an up-to-date insightful coverage to the recent advances in the synthesis, characterization, functional properties and potential device applications in specialized areas.

\title{
How to reference
}

In order to correctly reference this scholarly work, feel free to copy and paste the following:

Evgeniy Vilkov and Sergey Maryshev (2010). Electroacoustic Waves in a Ferroelectric Crystal with of a Moving System of Domain Walls, Ferroelectrics, Dr Indrani Coondoo (Ed.), ISBN: 978-953-307-439-9, InTech, Available from: http://www.intechopen.com/books/ferroelectrics/electroacoustic-waves-in-a-ferroelectriccrystal-with-of-a-moving-system-of-domain-walls

\section{INTECH}

open science | open minds

\section{InTech Europe}

University Campus STeP Ri

Slavka Krautzeka 83/A

51000 Rijeka, Croatia

Phone: +385 (51) 770447

Fax: +385 (51) 686166

www.intechopen.com

\section{InTech China}

Unit 405, Office Block, Hotel Equatorial Shanghai

No.65, Yan An Road (West), Shanghai, 200040, China

中国上海市延安西路65号上海国际贵都大饭店办公楼 405 单元

Phone: +86-21-62489820

Fax: +86-21-62489821 
(C) 2010 The Author(s). Licensee IntechOpen. This chapter is distributed under the terms of the Creative Commons Attribution-NonCommercialShareAlike-3.0 License, which permits use, distribution and reproduction for non-commercial purposes, provided the original is properly cited and derivative works building on this content are distributed under the same license. 